This is the peer reviewed version of the following article:

Isogeometric topology optimization for continuum structures using density distribution function

International Journal for Numerical Methods in Engineering.

First published: 21 April 2019

which has been published in final form at

https://doi.org/10.1002/nme.6081

This article may be used for non-commercial purposes in accordance with Wiley Terms and Conditions for Self-Archiving." 


\title{
Isogeometric topology optimization for continuum structures using density distribution function
}

\author{
Jie $\mathrm{Gao}^{1,2}$, Liang $\mathrm{GaO}^{2}$, *Zhen $\mathrm{Luo}^{1}$, Peigen $\mathrm{Li}^{2}$ \\ ${ }^{1}$ The School of Mechanical and Mechatronic Engineering, University of Technology Sydney, 15 Broadway, \\ Ultimo, NSW 2007, Australia \\ ${ }^{2}$ The State Key Lab of Digital Manufacturing Equipment and Technology, Huazhong University of Science and \\ Technology, 1037 Luoyu Road, Wuhan, Hubei 430074, China \\ * Corresponding author: Tel.: +61-2-95142994; E-mail: zhen.luo@uts.edu.au (A/Prof Z. Luo)
}

\begin{abstract}
This paper will propose a more effective and efficient topology optimization method based on isogeometric analysis, termed as isogeometric topology optimization (ITO), for continuum structures using an enhanced density distribution function (DDF). The construction of the DDF involves two steps: (1) Smoothness: the Shepard function is firstly utilized to improve the overall smoothness of nodal densities. Each nodal density is assigned to a control point of the geometry; (2) Continuity: the high-order NURBS basis functions are linearly combined with the smoothed nodal densities to construct the DDF for the design domain. The nonnegativity, partition-of-unity and restricted bounds $[0,1]$ of both the Shepard function and NURBS basis functions can guarantee the physical meaning of material densities in the design. A topology optimization formulation to minimize the structural mean compliance is developed based on the DDF and isogeometric analysis (IGA) to solve structural responses. An integration of the geometry parametrization and numerical analysis can offer the unique benefits for the optimization. Several 2D and 3D numerical examples are performed to demonstrate the effectiveness and efficiency of the proposed ITO method, and the optimized 3D designs are prototyped using the Selective Laser Sintering (SLS) technique.
\end{abstract}

Keywords: Topology optimization; Isogeometric analysis; Density distribution function; NURBS. 


\section{Introduction}

In recent years, topology optimization has accepted enormous attention and considerable developments due to its capability in finding the optimal material layout in the conceptual design stage of products [1]. Many topology optimization methods have been proposed, such as the homogenization method [2], the Solid Isotropic Material with Penalization (SIMP) method [3,4], the Evolutionary Structural Optimization (ESO) method [5], the Level Set Method (LSM) [6-8] and the Moving Morphable Components (MMC) [9,10], with a wide range of applications, including the frequency responses [11-13], material microstructures [14$16]$ and multiscale design [17-24], as well as stress problems [25,26] and etc [27].

Material description models (MDMs) have been widely adopted to describe the structural topology due to its easiness [2-5]. SIMP can be regarded as a powerful variant of the homogenization method, which has received much popularity due to its conceptual simplicity $[3,4]$. It is noticed that the element densities act as design variables in the classic SIMP-based approaches. The finite elements are employed not only in the numerical analysis to solve the structural responses, but also represent the material layout [1]. The topology optimization to search the continuous material distribution is fully converted into the spatial arrangement of elemental densities. The numerical artifacts are often occurred in designs, including the checkerboards, “zig-zag" structural boundaries and mesh-dependency [28-30]. Several works reveal that these numerical issues may be because of the low order finite elements used in the SIMP method [31-33].

As a variant of the classic SIMP method, nodal density-based design variables have been introduced in the topology optimization with a view to eliminating the numerical artifacts. Matsui and Terada [31] proposed a CAMD (the continuous approximation of material distribution) method to emphasis the material density field in the structural design domain. A Q4/Q4 numerical implementation to maintain the $\mathrm{C}^{0}$ continuity of the design variables in the SIMP model is presented to suppress the checkerboard pattern [32]. However, an important numerical phenomenon, namely the "islanding" or "layering" structures, is occurred in the optimized solutions. After that, Paulino et al [33] rearranged the design variables in Q4 elements to avoid the "islanding" phenomenon by an internal averaging technique. Guest et al [34] also introduced the nodal design variables and a projection scheme to control the minimum length in the designs. Kang and Wang $[35,36]$ developed the interpolation scheme using the Shepard function to eliminate the "islanding" and "layering" structures. Later, Luo et al [37] proposed a dual-level density interpolation using the Shepard function based on the element-free Galerkin method, which can effectively remove the numerical issues in the finite elements-based SIMP methods. However, the computational efficiency of the meshless methods 
limited the application to three-dimensional designs. It can be concluded that the distribution of densities in the design domain is expressed by a interpolation function developed by the Lagrange polynomials [3137]. However, it should be noted that the oscillatory of the Lagrange polynomials might has an influence on the smoothness and continuity of the density distribution.

On the other side, the numerical analysis in most current topology optimization methods is dominated by the finite element method (FEM). In topology optimization, the finite element method is featured with the following limitations: (1) The finite element mesh cannot exactly capture the structural geometry, which lower the numerical precision [38,39]; (2) The lower-order $\left(\mathrm{C}^{0}\right)$ continuity of the structural responses in FEM affects the accuracy of the optimized results; (3) To achieve a high quality of the finite element mesh is not easy. IGA, proposed by Hughes and his co-workers [38,39], can be regarded as an extension and generalization of the conventional finite element methods to perform the numerical analysis. The idea of IGA is to employ the same basis functions (e.g. NURBS) to represent both the geometry parametrization and the finite dimensional solution space in the numerical analysis, so that the geometrical model and the numerical analysis model can be consistent [40].

As far as the application of IGA into topology optimization, the first work might be [41], where the trimmed spline surfaces were applied to present the structural boundaries. Later, Dedè et al. [42] developed a phase field method with IGA for the topology optimization of continuum structures. Hassani et al. [43] proposed an isogeometric approach for the topology optimization, where a continuous material distribution function was developed using the NURBS. Hassani et al. [44] also addressed the simultaneous shape and topology optimization for shell structures, using NURBS to model free form shells. The trimmed NURBS surfaces were also applied in the isogeometric topology optimization of shell structures [45]. A B-spline space with the intrinsic filter for topology optimization was discussed in [46]. The IGA was also introduced into the parametric level set method $[47,48]$ and developed a level-set based topology optimization method with IGA [48], where the level set function was interpolated by the NURBS basis functions, rather than using the compactly supported radial basis functions given in [46,47]. In [50], an isogeometric approach was also developed for the level set topology optimization, where three different problems were discussed, like the minimizing weight subject to the local stress constraints. Topology optimization with the global stress constraint was also studied in an IGA-based SIMP framework [51]. The level-set based IGA formulation for topology optimization of flexoelectric materials was developed [52], and the multi-resolution topology optimization problem using IGA was also discussed in [53]. As we can see, the IGA-based level set methods 
have been discussed for the topology optimization of structures to a great extent. Only a limited number of works are devoted to study the positive features of the IGA applied into MDMs.

The core of the current work is to construct an enhanced DDF with the sufficient smoothness and continuity for the development of a new isogeometric topology optimization (ITO) method, that is to be more effective and efficient. The ITO method will be applied to optimize not only 2D structures with the rectangle, curved and complex design domains, but also the 3D scenario. Meanwhile, the SLS technique is used to fabricate the optimized 3D designs, which demonstrates the benefits of the additive manufacture for the realization of topologically optimized results. In the development of the DDF, the Shepard function is firstly applied to improve the overall smoothness of the nodal densities at control points, and the DDF is then constructed by a linear combination of NURBS basis functions with the smoothed nodal densities. Hence, the same NURBS basis functions are used to construct both IGA and the DDF. IGA and DDF are further applied to develop the ITO formulation for minimizing the structural mean compliance. The core of the ITO method aims to optimize the densities in the DDF within a given design domain, in order to optimize the structural performance that is relevant to the DDF.

\section{NURBS-based IGA}

IGA is a recently proposed computational approach that offers the possibility of integrating the numerical analysis and computer aided design into a single unified framework [38,39]. A large number of candidate computational geometry technologies can be applied into IGA, in which the most widely used are NURBS due to the major strengths to exactly model a wide array of objects [54].

\subsection{NURBS basis functions}

In one dimension for the B-spline curve, a knot vector with an ordered set of increasing parameters must be defined in the parametric space, written $\Xi=\left\{\xi_{1}, \xi_{2}, \cdots, \xi_{n+p+1}\right\}$, where $\xi_{i} \in \mathbb{R}\left(\xi_{i} \leq \xi_{i+1}\right)$ is the $i^{\text {th }}$ knot, $n$ is the number of basis functions to build the B-spline curve and $p$ is the polynomial order. The interval $\left[\xi_{1}, \xi_{n+p+1}\right]$ is called a patch in the parametric space. The knot vector partitions the parametric space into a series of sub intervals, generally referred to knot spans. The B-spline basis functions are defined recursively by the Cox-de-Boor formula [54], starting with piecewise constants $(p=0)$ :

$$
N_{i, 0}(\xi)= \begin{cases}1 & \text { if } \xi_{i} \leq \xi_{i+1} \\ 0 & \text { otherwise }\end{cases}
$$

For $p \geq 1$, the basis functions are defined by 


$$
N_{i, p}(\xi)=\frac{\xi-\xi_{i}}{\xi_{i+p}-\xi_{i}} N_{i, p-1}(\xi)+\frac{\xi_{i+p+1}-\xi}{\xi_{i+p+1}-\xi_{i+1}} N_{i+1, p-1}(\xi)
$$

In Eq. (2), fractions with the form 0/0 are defined as zero. It can be observed that the basis functions with $p=0,1$ defined in Eqs. (1) and (2) are same as the standard piecewise constant and linear finite element functions, respectively. However, functions with the higher order $(p \geq 2)$ are completely different. Some important properties of the B-spline basis functions are listed as follows:

(1) Nonnegativity: $N_{i, p}(\xi) \geq 0$.

(2) Local support: the support of each basis function $N_{i, p}$ is contained in the interval $\left[\xi_{i}, \xi_{i+p+1}\right]$. Also, at most $p+1$ number of B-spline basis functions are nonzero in the given knot span $\left[\xi_{i}, \xi_{i+1}\right]$.

(3) Partition of unity: For an arbitrary knot $\operatorname{span}\left[\xi_{i}, \xi_{i+1}\right], \forall \xi \in\left[\xi_{i}, \xi_{i+1}\right], \sum_{j=i-p}^{i} N_{j, p}(\xi)=1$.

(4) Continuity: The continuity between knot spans is $C^{p-k}$ where $k$ is the multiplicity of knots.

Although B-splines are flexible for modeling many geometries, some important shapes are not represented, like the circles and ellipsoids. Hence, NURBS acting as a generalization form of B-splines is defined, where the NURBS basis function is formed by assigning a positive weight $\omega_{i}$ to B-spline basis function, as:

$$
R_{i, p}(\xi)=\frac{N_{i, p}(\xi) \omega_{i}}{\sum_{j=1}^{n} N_{j, p}(\xi) \omega_{j}}
$$

In two and three dimensions, NURBS basis functions can be constructed as:

$$
\left\{\begin{array}{l}
R_{i, j}^{p, q}(\xi, \eta)=\frac{N_{i, p}(\xi) M_{j, q}(\eta) \omega_{i j}}{\sum_{\hat{\imath}=1}^{n} \sum_{\hat{\jmath}=1}^{m} N_{\hat{\imath}, p}(\xi) M_{\hat{\jmath}, q}(\eta) \omega_{\hat{\imath} \hat{\jmath}}} \\
R_{i, j, k}^{p, q, r}(\xi, \eta, \zeta)=\frac{N_{i, p}(\xi) M_{j, q}(\eta) L_{k, r}(\zeta) \omega_{i j k}}{\sum_{\hat{\imath}=1}^{n} \sum_{\hat{\jmath}=1}^{m} \sum_{\hat{k}=1}^{l} N_{\hat{\imath}, p}(\xi) M_{\hat{\jmath}, q}(\eta) L_{\hat{k}, r}(\zeta) \omega_{\hat{\imath} \hat{\jmath} \hat{k}}}
\end{array}\right.
$$

where $M_{j, q}$ and $L_{k, r}$ are B-spline basis functions in other two parametric directions, constructed by knot vectors $\mathcal{H}=\left\{\eta_{1}, \eta_{2}, \cdots, \eta_{m+q+1}\right\}$ and $Z=\left\{\zeta_{1}, \zeta_{2}, \cdots, \zeta_{l+r+1}\right\}$, respectively. $q$ and $r$ are the polynomial orders in two parametric directions, respectively, and $m$ and $l$ are the corresponding numbers. NURBS basis functions can inherit all the favorable properties of B-spline basis functions.

\subsection{Geometry parametrization for structural design domain}

As displayed in Fig. 1, a 3D Bridge-type structure is modeled by 3D NURBS basis functions with a set of the control points. The structural domain is shown in Fig. 1 (a), and the NURBS-based geometrical model is displayed in Fig. $1(b)$. The IGA mesh for the numerical analysis is given in Fig. $1(c)$. The NURBS basis functions in three parametric directions are displayed in Fig. $1(d),(e)$ and $(f)$, respectively. Given a control lattice $\mathbf{P}_{i, j, k} \in \mathbb{R}^{3}$, a tensor product NURBS solid $\mathbf{S}(\xi, \eta, \zeta)$ is defined, as: 


$$
\mathbf{S}(\xi, \eta, \zeta)=\sum_{i=1}^{n} \sum_{j=1}^{m} \sum_{k=1}^{l} R_{i, j, k}^{p, q, r}(\xi, \eta, \zeta) \mathbf{P}_{i, j, k}
$$

As already pointed outed in [38,39], NURBS has some important properties, namely 1) Strong convex hull property; 2) Differentiability; 3) Local modification; 4) Variation diminishing property. The properties are important for the later construction of the DDF. In Fig. $1(a)$, it can be easily seen that the structure can be exactly represented by the NURBS basis functions with control points. The knot vectors partition the whole patch in the parametric space into an array of knot spans, also called elements. Element boundaries in the physical space correspond to the images of knot lines in the NURBS mapping. In order to distinguish the elements in FEM, the knot spans are named by "IGA elements". The IGA mesh is also presented Fig. 1 (c). As displayed in Fig. $1(b)$ and $(c)$, the control points do not coincide with the nodes in IGA elements. The IGA mesh model is consistent with the geometrical model of the 3D Bridge-type structure.

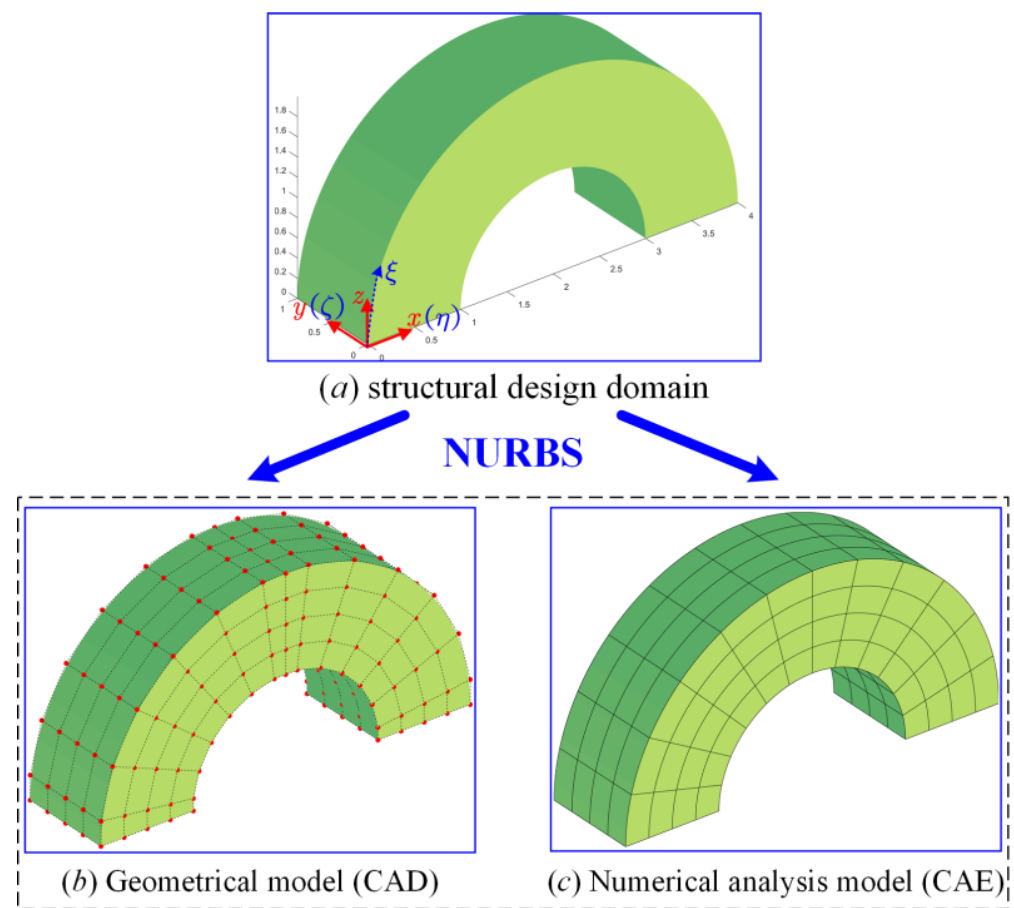

INTEGRATION of CAD and CAE

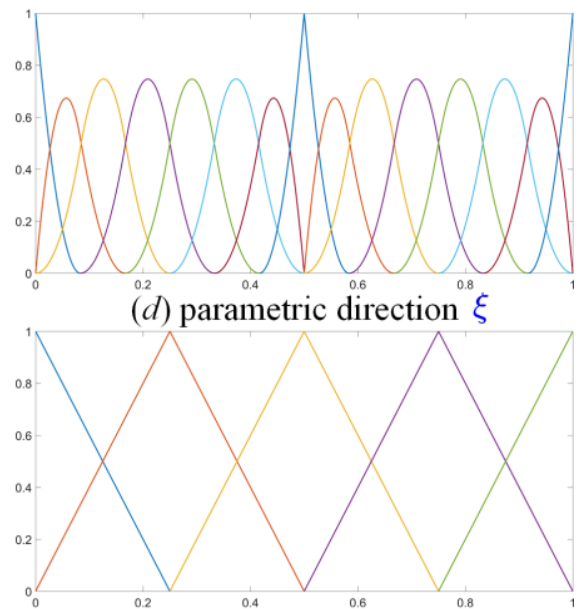

$\stackrel{02}{(e)}$ parametric direction ${ }^{0.8} \eta$

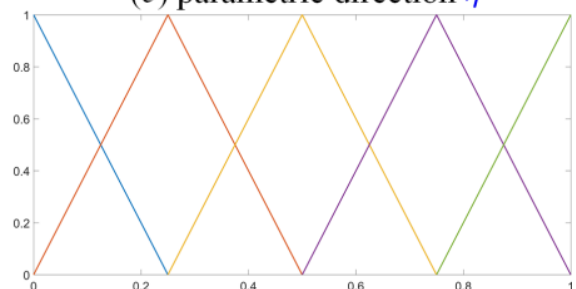

$(f)$ parametric direction $\zeta$

Fig. 1. NURBS-based IGA for 3D Bridge-type structure: $\Xi=\{0,0,0,0.0833, \cdots, 0.9167,1,1,1\}, \mathcal{H}=$ $\{0,0,0.25,0.5,0.75,1,1\}, Z=\{0,0,0.25,0.5,0.75,1,1\} ; n=15, m=l=5 ; p=2, q=r=1$.

\subsection{Galerkin's Formulation for elastostatics}

As an example of application of the NURBS-based IGA, the linear elasticity is considered only for the sake of numerical simplicity but without losing any generality. A strong form of the boundary value problem for a design domain $\Omega$ bounded by the boundary $\Gamma$ is formally stated. Given $f_{i}: \Omega \rightarrow \mathbb{R}, g_{i}: \Gamma_{D_{i}} \rightarrow \mathbb{R}$ and $h_{i}: \Gamma_{N_{i}} \rightarrow \mathbb{R}$ find $u_{i}: \bar{\Omega} \rightarrow \mathbb{R}$ such that 


$$
\left\{\begin{array}{cc}
\sigma_{i j, j}+f_{i}=0 & \text { in } \Omega \\
u_{i}=g_{i} & \text { on } \Gamma_{D_{i}} \\
\sigma_{i j} n_{j}=h_{i} & \text { on } \Gamma_{N_{i}}
\end{array}\right.
$$

where $\sigma$ is stress tensor, $f_{i}$ is the body force, and $g_{i}$ is the prescribed boundary displacement on the Dirichlet boundary $\Gamma_{D} . h_{i}$ is the boundary traction on the Neumann boundary $\Gamma_{N} \cdot n_{j}$ is the component of the unit normal vector to $\Omega$. Defining a trial solution space $\mathcal{S}=\left\{\mathbf{u} \mid u_{i} \in \mathcal{S}_{i}\right\}$ and a weight space by $\mathcal{V}=\left\{\mathbf{w} \mid w_{i} \in \mathcal{V}_{i}\right\}$, where each trial solution $u_{i}$ satisfies the Dirichlet condition $u_{i}=g_{i}$ on $\Gamma_{D_{i}}$ and each weight function $w_{i}$ is equal to 0 on the Dirichlet boundary $\Gamma_{D_{i}}$. Given $\mathbf{f}=\left\{f_{i}\right\}, \mathbf{g}=\left\{g_{i}\right\}$ and $\mathbf{h}=\left\{h_{i}\right\}$, find $\mathbf{u} \in \mathcal{S}$ such that for all $\mathbf{w} \in \mathcal{V}$,

$$
\int_{\Omega} \boldsymbol{\varepsilon}(\mathbf{w})^{T} \mathbf{D} \boldsymbol{\varepsilon}(\mathbf{u}) d \Omega=\int_{\Omega} \mathbf{f} \mathbf{w} d \Omega+\int_{\Gamma_{N}} \mathbf{h w} d \Gamma_{N}
$$

where $\boldsymbol{\varepsilon}$ is the strain, and $\mathbf{D}$ is the elastic tensor matrix. In the Galerkin's method, the finite-dimensional approximants of the spaces $\mathcal{S}$ and $\mathcal{V}$ are also constructed by NURBS basis functions, denoted by $\mathcal{S}^{h}$ and $\mathcal{V}^{h}$, which consists of all linear combinations of the NURBS basis functions. The finite-dimensional nature of the function converts the weak form of the problem into a system of linear algebraic equations, and Eq. (7) can be stated as a form:

$$
\int_{\Omega} \boldsymbol{\varepsilon}\left(\mathbf{w}^{h}\right)^{T} \mathbf{D} \boldsymbol{\varepsilon}\left(\mathbf{v}^{h}\right) d \Omega=\int_{\Omega} \mathbf{f} \mathbf{w}^{h} d \Omega+\int_{\Gamma_{N}} \mathbf{h} \mathbf{w}^{h} d \Gamma_{N}-\int_{\Omega} \boldsymbol{\varepsilon}\left(\mathbf{w}^{h}\right)^{T} \mathbf{D} \boldsymbol{\varepsilon}\left(\mathbf{g}^{h}\right) d \Omega
$$

Introducing a set containing all NURBS basis functions, denoted by $\mathcal{A}$, and a sub set containing the basis functions that are equal to 0 on the Dirichlet boundary $\Gamma_{D}$, symbolled by $\mathcal{B}(\mathcal{B} \subset \mathcal{A})$. The trial solution $\mathbf{u}^{h} \in \mathcal{S}^{h}$ and weight $\mathbf{w}^{h} \in \mathcal{V}^{h}$ can be stated as a function of the basis functions with control variables:

$$
\left\{\begin{array}{l}
\mathbf{u}^{h}=\sum_{j \in \mathcal{A} \backslash \mathcal{B}} R_{j} g_{i}+\sum_{i \in \mathcal{B}} R_{i} d_{i} \\
\mathbf{w}^{h}=\sum_{j \in \mathcal{A} \backslash \mathcal{B}} R_{j} c_{i}
\end{array}\right.
$$

where $c$ is the arbitrary to hold for all $\mathbf{w}^{h} \in \mathcal{V}^{h}$. Substituting Eq. (9) into Eq. (8), the Galerkin form is transformed into the next equation, given as:

$$
\left(\int_{\Omega} \boldsymbol{\varepsilon}\left(R_{i}\right)^{T} \mathbf{D} \boldsymbol{\varepsilon}\left(R_{j}\right) d \Omega\right) d_{i}=\int_{\Omega} \mathbf{f} R_{j} d \Omega+\int_{\Gamma_{N}} \mathbf{h} R_{j} d \Gamma_{N}-\int_{\Omega} \boldsymbol{\varepsilon}\left(R_{j}\right)^{T} \mathbf{D} \boldsymbol{\varepsilon}\left(g^{h}\right) d \Omega
$$

Proceeding to define 


$$
\left\{\begin{array}{l}
K_{i j}=\int_{\Omega} \boldsymbol{\varepsilon}\left(R_{i}\right)^{T} \mathbf{D} \boldsymbol{\varepsilon}\left(R_{j}\right) d \Omega \\
F_{j}=\int_{\Omega}^{\mathbf{f}} R_{j} d \Omega+\int_{\Gamma_{N}} \mathbf{h} R_{j} d \Gamma_{N}-\int_{\Omega} \boldsymbol{\varepsilon}\left(R_{j}\right)^{T} \mathbf{D} \boldsymbol{\varepsilon}\left(g^{h}\right) d \Omega
\end{array}\right.
$$

Hence, Eq. (10) can be expanded a more compact matrix form, as:

$$
\mathbf{K d}=\mathbf{F}
$$

where,

$$
\mathbf{K}=\left[K_{i j}\right] ; \mathbf{d}=\left\{d_{i}\right\} ; \mathbf{F}=\left\{F_{j}\right\}
$$

In IGA, the NURBS basis functions is firstly applied to model the exact geometry, and then discretize the structural geometry into a series of IGA elements, as well as serving as the basis functions to construct the solution space and the weight space. Hence, the NURBS basis functions can perfectly unify the geometry construction, spatial discretization and numerical analysis into a single framework.

\section{Density distribution function (DDF)}

In most element-based MDMs, the problem of the material distribution has been transformed to the spatial arrangement of finite elements. The structural topology is expressed by the spatial arrangement of discrete finite elements. Two basic requirements must be satisfied to ensure a justified density distribution [35-37]: 1) The nonnegativity of element densities; 2) The strict bounds of element densities, namely $[0,1]$.

This section focuses on constructing an enhanced DDF with the sufficient smoothness and continuity using the Shepard function and NURBS basis functions. Firstly, the Shepard function is applied to enhance the overall smoothness of nodal densities at control points. Secondly, the NURBS basis functions are linearly combined with the preciously smoothed nodal densities to construct the DDF. In this way, both the DDF and the geometry parametrization of the structural design domain are constructed with the same NURBS basis functions. The DDF can be viewed as a response surface with a higher dimension with respect to the nodal densities, which is used to represent the density distribution for the design domain.

\subsection{Smooth nodal densities using the Shepard function}

In Fig. 2, a cross-sectional view of the IGA mesh for the 3D Bridge-type structure is presented, including the Gauss quadrature points plotted with the black, the control points shown by the red and green dots and the IGA elements bounded by black lines. In the NURBS solid, each control point is assigned by a nodal density, and a lattice of nodal densities at control points is defined. In the DDF, the overall smoothness of 
nodal densities should be improved to make sure the sufficient smoothness of the DDF. The basic principle is that each nodal density is equal to the mean value of all nodal densities located at the local support area of the current nodal density, as shown by the purple circular area in Fig. 2 (a), as:

$$
\mathcal{G}\left(\rho_{i, j, k}\right)=\sum_{i=1}^{\mathcal{N}} \sum_{j=1}^{\mathcal{M}} \sum_{k=1}^{\mathcal{L}} \psi\left(\rho_{i, j, k}\right) \rho_{i, j, k}
$$

where $\mathcal{G}$ is the smoothed nodal density at the $(i, j, k)$ control point, and $\rho_{i, j, k}$ is the initial nodal density which should maintain the nonnegative and the range-bounded by 0 and $1 . \mathcal{N}, \mathcal{M}$ and $\mathcal{L}$ are the numbers of nodal densities located at the local support area of the current nodal density in three parametric directions, respectively. $\psi\left(\rho_{i, j, k}\right)$ is the value of the Shepard function at the nodal $(i, j, k)$, and expressed as [55]:

$$
\psi\left(\rho_{i, j, k}\right)=\frac{w\left(\rho_{i, j, k}\right)}{\sum_{\hat{\imath}=1}^{\mathcal{N}} \sum_{\hat{\jmath}=1}^{\mathcal{M}} \sum_{\hat{k}=1}^{\mathcal{L}} w\left(\rho_{\hat{\imath}, \hat{\jmath}, \hat{k}}\right)}
$$

where $w$ is the weight function of the $(i, j, k)$ nodal density, and it can be constructed by many functions, like the inverse distance weighting function, the exponential cubic spline, quartic spline functions and radial basis functions (RBFs) $[46,47,55]$. The compactly supported RBFs (CSRBFs) with the $\mathrm{C}^{4}$ continuity are used due to the compactly supported, the high-order continuity and the nonnegativity, by:

$$
w(r)=(1-r)_{+}^{6}\left(35 r^{2}+18 r+3\right)
$$

where $r=d / d_{m}$, and $d$ is the Euclidean distance between the current nodal density and the other nodal density located at the local support domain. $d_{m}$ is the radius of the local support domain which indicates the scale of the local support domain, as shown in Fig. $2(a)$. It can be seen that the smoothed nodal densities by the Shepard function can meet the necessary conditions for a physically meaningful material density: 1) Non-negativity 2) Strict-bounds [0, 1]. It is also important to notice that the Shepard function for the nodal densities is not a processing procedure as a heuristic filtering scheme, and it will be considered in the next optimization formulation.

\subsection{NURBS for the construction of the DDF}

The NURBS basis functions are linearly combined with the smoothed nodal densities to construct the DDF. Assuming that the DDF in the structural design domain is denoted by $\chi(\xi, \eta, \zeta)$, given as:

$$
\mathcal{X}(\xi, \eta, \zeta)=\sum_{i=1}^{n} \sum_{j=1}^{m} \sum_{k=1}^{l} R_{i, j, k}^{p, q, r}(\xi, \eta, \zeta) \mathcal{G}\left(\rho_{i, j, k}\right)
$$


It can be seen that Eq. (17) for the DDF has the same mathematical formulation for NURBS solid in Eq. (5). The key difference is located at the physical meanings of control coefficients. In Eq. (5), the control coefficients correspond to the physical coordinates of control points, but the coefficients in Eq. (17) are the physical densities on control points. Hence, the geometry parametrization using the NURBS solid for the 3D Bridge-type structure is converted into a physical representation by the DDF for the design domain. Eq. (17) is the global form of the DDF. This form can be expanded as a local form depended on the local support domain of the current knot $(\xi, \eta, \zeta) \in\left[\xi_{i}, \xi_{i+1}\right] \times\left[\eta_{j}, \eta_{j+1}\right] \times\left[\zeta_{k}, \zeta_{k+1}\right]$, that

$$
\mathcal{X}(\xi, \eta, \zeta)=\sum_{e=i-p}^{i} \sum_{f=j-q}^{j} \sum_{g=k-r}^{k} R_{e, f, g}^{p, q, r}(\xi, \eta, \zeta) \mathcal{G}\left(\rho_{e, f, g}\right)
$$

It can be easily seen that the properties 1 to 3 of the NURBS basis functions described in Section 2.1 ensure the DDF with two properties: 1) the Nonnegativity; 2) Strict bounds. Hence, the DDF in Eq. (18) can be featured with the strict physical meanings in the next optimization formulation. It is important to note that the NURBS basis functions do not satisfy the interpolation property, which has no influence on the DDF. The main reason is that control points are not necessary on the structural design domain. The nodal densities only work as control coefficients in the combination with the NURBS basis functions to construct the DDF. This is the key difference compared to the previous works where material density functions are developed by Lagrange polynomials [31-37]. Meanwhile, the variation diminishing property of NURBS can remove the oscillatory of Lagrange polynomials [38,39], which ensure the sufficient smoothness and continuity of the DDF. Inspired by [43], the current work also employs NURBS to construct material density distribution function, but its smoothness and continuity are improved. Hence, the DDF will be more beneficial to the latter development of the ITO formulation.

In the Galerkin IGA formulation, the system stiffness matrix and load vector are achieved by assembling the local stiffness matrix and load vector, respectively. The design domain is discretized by a series of IGA elements. The evaluation of the IGA element stiffness matrix is performed by the Gauss quadrature. In the physical space, the IGA element stiffness matrix and force vector are calculated by:

$$
\left\{\begin{array}{l}
\mathbf{K}_{e}=\int_{\Omega_{e}} \mathbf{B}^{T} \mathbf{D B} d \Omega_{e} \\
\mathbf{F}_{e}=\int_{\Omega_{e}} \mathbf{f} R d \Omega_{e}+\int_{\Gamma_{N}^{e}} \mathbf{h} R d \Gamma_{N}^{e}-\mathbf{K}_{e} \mathbf{g}_{e}
\end{array}\right.
$$


where $\Omega_{e}$ is the physical domain of the IGA element, as shown in Fig. 2, $\Gamma_{N}^{e}$ is the Neumann boundary condition of the IGA element and $\mathbf{g}_{e}$ is the Dirichlet boundary condition. $\mathbf{B}$ is the strain-displacement matrix calculated by partial derivatives of NURBS basis functions, as given in Eq. (11). The iso-parametric formulation has been employed to evaluate the element stiffness matrix. As presented in Fig. 2, a mapping $\mathbf{X}: \widehat{\Omega}_{e} \rightarrow \Omega_{e}$ from the parametric space to the physical space and an affine mapping $\mathbf{Y}: \widetilde{\Omega}_{e} \rightarrow \widehat{\Omega}_{e}$ from the bi-unit parent element to the element in the parametric space are defined. The integral for the IGA element stiffness matrix is pulled back first onto the parametric element and then onto the bi-unit parent element, which contains the inverses of the two mappings, by:

$$
\mathbf{K}_{e}=\int_{\widetilde{\Omega}_{e}} \mathbf{B}^{T} \mathbf{D B}\left|J_{1}\right|\left|J_{2}\right| d \widetilde{\Omega}_{e}
$$

where $\boldsymbol{J}_{1}$ and $\boldsymbol{J}_{2}$ are Jacobi matrices of the two mappings, respectively. As shown in Fig. 2, all the Gauss quadrature points in the IGA mesh and a $3 \times 3$ of the Gauss quadrature points in each IGA element are both displayed, which are denoted by the black dots. The detailed numerical computation of the element stiffness matrix is expressed by:

$$
\mathbf{K}_{e}=\sum_{i=1}^{3} \sum_{j=1}^{3} \sum_{k=1}^{3}\left\{\mathbf{B}^{T}\left(\xi_{i}, \eta_{j}, \zeta_{k}\right) \mathbf{D B}\left(\xi_{i}, \eta_{j}, \zeta_{k}\right)\left|\boldsymbol{J}_{1}\left(\xi_{i}, \eta_{j}, \zeta_{k}\right)\right|\left|\boldsymbol{J}_{2}\left(\xi_{i}, \eta_{j}, \zeta_{k}\right)\right| \omega_{i} \omega_{j} \omega_{k}\right\}
$$

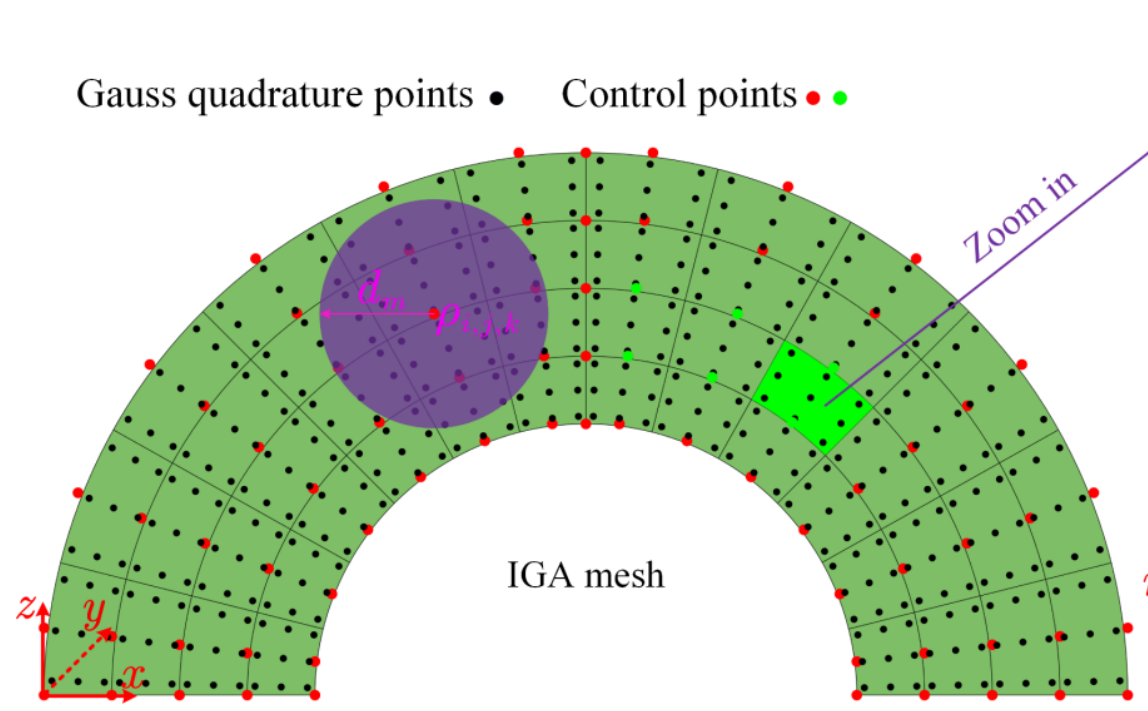

(a) Cross-sectional view of geometrical model (Physical space)

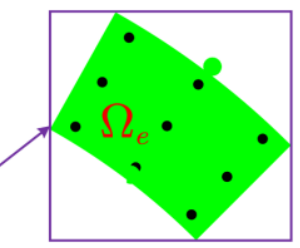

IGA element Bi-unit parent element

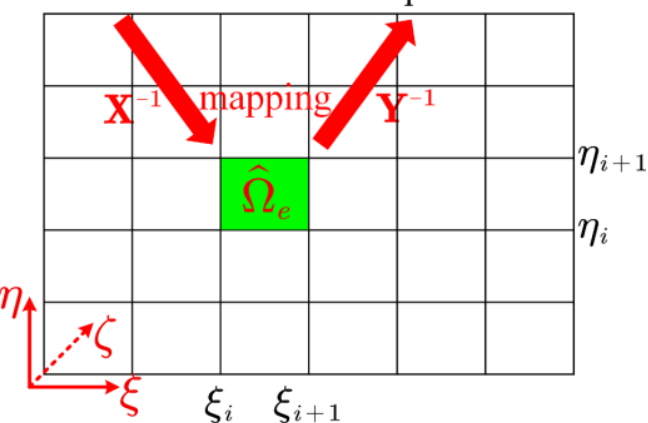

(b) Parametric space

Fig. 2. Cross-sectional view of the IGA mesh for the 3D Bridge-type structure

where $\omega_{i}, \omega_{j}$ and $\omega_{k}$ are the corresponding quadrature weights. In MDMs, the elasticity property of the isotropic material is assumed to be a power function of material density with a penalty parameter. Hence, the IGA element stiffness matrix should be expressed by a function of the densities at the Gauss quadrature points, rather than the constant element density [31-36], as follows: 


$$
\mathbf{K}_{e}=\sum_{i=1}^{3} \sum_{j=1}^{3} \sum_{k=1}^{3}\left\{\mathbf{B}^{T}\left(\xi_{i}, \eta_{j}, \zeta_{k}\right)\left(x\left(\xi_{i}, \eta_{j}, \zeta_{k}\right)\right)^{\gamma} \mathbf{D}_{0} \mathbf{B}\left(\xi_{i}, \eta_{j}, \zeta_{k}\right)\left|\boldsymbol{J}_{1}\right|\left|\boldsymbol{J}_{2}\right| \omega_{i} \omega_{j} \omega_{k}\right\}
$$

where $\mathcal{X}$ is the density of the $\left(\xi_{i}, \eta_{j}, \zeta_{k}\right)$ Gauss quadrature point, and $\gamma$ is the penalization parameter. $\mathbf{D}_{0}$ is the elastic tensor matrix for the solid density.

\section{Isogeometric topology optimization (ITO)}

\subsection{ITO formulation to minimize static compliance}

In this Section, the DDF is adopted to develop the ITO formulation, with the IGA applied to calculate the related structural responses. Hence, the ITO formulation will iteratively optimize the density distribution represented by the DDF in the design domain, until the topology has the optimal stiffness performance. As already given in Section 3, nodal densities are control coefficients in the DDF. Here, nodal densities work as design variables at here to evolve the DDF during the optimization, given as:

$$
\left\{\begin{array}{l}
\text { Find: } \rho_{i, j, k}(i=1,2, \cdots, n ; j=1,2, \cdots, m ; k=1,2, \cdots, l) \\
\text { Min: } J(\mathbf{u}, \mathcal{X})=\frac{1}{2} \int_{\Omega} \boldsymbol{\varepsilon}(\mathbf{u})^{T} \mathbf{D}(X(\xi, \eta, \zeta)) \boldsymbol{\varepsilon}(\mathbf{u}) d \Omega \\
\text { S.t: }\left\{\begin{array}{l}
G(X)=\frac{1}{|\Omega|} \int_{\Omega} X(\xi, \eta, \zeta) v_{0} d \Omega-V_{0} \leq 0 \\
a(\mathbf{u}, \delta \mathbf{u})=l(\delta \mathbf{u}),\left.\mathbf{u}\right|_{\Gamma_{D}}=\mathbf{g}, \forall \delta \mathbf{u} \in H^{1}(\Omega) \\
0 \leq \rho_{i, j, k} \leq 1
\end{array}\right.
\end{array}\right.
$$

where $\rho_{i, j, k}$ is the initial nodal densities at control points, and $J$ is the objective function defined by the structural mean compliance. $G$ is the volume constraint. $v_{0}$ is the volume fraction of solids and $V_{0}$ is the maximum material consumption. $\mathbf{u}$ is the unknown displacement field in the design domain $\Omega$, and $\mathbf{g}$ is the prescribed displacement vector on the Dirichlet boundary $\Gamma_{D} . \delta \mathbf{u}$ is the virtual displacement field belonging to the kinematically admissible space $H^{1}(\Omega)$. $a$ and $l$ are the bilinear energy and linear load functions, respectively, defined by:

$$
\left\{\begin{array}{l}
a(\mathbf{u}, \delta \mathbf{u})=\int_{\Omega} \boldsymbol{\varepsilon}(\mathbf{u})^{T} \mathbf{D}(\chi(\xi, \eta, \zeta)) \boldsymbol{\varepsilon}(\delta \mathbf{u}) d \Omega \\
l(\delta \mathbf{u})=\int_{\Omega} \mathbf{f} \delta \mathbf{u} d \Omega+\int_{\Gamma_{N}} \mathbf{h} \delta \mathbf{u} d \Gamma_{N}
\end{array}\right.
$$

where $\mathbf{f}$ is the body force and $\mathbf{h}$ is the boundary traction on the Neumann boundary $\Gamma_{N}$.

\subsection{Design Sensitivity analysis}

In Eq. (23), the first-order derivative of the objective function with respect to the DDF is firstly given by: 


$$
\frac{\partial J}{\partial \chi}=\int_{\Omega} \boldsymbol{\varepsilon}(\dot{\mathbf{u}})^{T} \mathbf{D}(\mathcal{X}(\xi, \eta, \zeta)) \boldsymbol{\varepsilon}(\mathbf{u}) d \Omega+\frac{1}{2} \int_{\Omega} \boldsymbol{\varepsilon}(\mathbf{u})^{T} \frac{\partial \mathbf{D}(\mathcal{X})}{\partial \mathcal{X}} \boldsymbol{\varepsilon}(\mathbf{u}) d \Omega
$$

where $\dot{\mathbf{u}}$ is the derivative of the displacement field with respect to the DDF. Performing the derivative on both sides of the equilibrium state equation given in Eq. (24), given by:

$$
\begin{aligned}
& \left\{\frac{\partial a}{\partial \chi}=\int_{\Omega} \boldsymbol{\varepsilon}(\dot{\mathbf{u}})^{T} \mathbf{D} \boldsymbol{\varepsilon}(\delta \mathbf{u}) d \Omega+\int_{\Omega} \boldsymbol{\varepsilon}(\mathbf{u})^{T} \mathbf{D} \boldsymbol{\varepsilon}(\delta \dot{\mathbf{u}}) d \Omega+\int_{\Omega} \boldsymbol{\varepsilon}(\mathbf{u})^{T} \frac{\partial \mathbf{D}(\mathcal{X})}{\partial \chi} \boldsymbol{\varepsilon}(\delta \mathbf{u}) d \Omega\right. \\
& \frac{\partial l}{\partial \chi}=\int_{\Omega} \mathbf{f} \delta \dot{\mathbf{u}} d \Omega+\int_{\Gamma_{N}} \mathbf{h} \delta \dot{\mathbf{u}} d \Gamma_{N}
\end{aligned}
$$

where $\delta \dot{\mathbf{u}}$ is the derivative of the virtual displacement field with respect to the DDF $\mathcal{X}$. Considering that $\delta \dot{\mathbf{u}} \in H^{1}(\Omega)$, the corresponding equilibrium state equation is given as:

$$
\int_{\Omega} \boldsymbol{\varepsilon}(\mathbf{u})^{T} \mathbf{D}(\mathcal{X}) \boldsymbol{\varepsilon}(\delta \dot{\mathbf{u}}) d \Omega=\int_{\Omega} \mathbf{f} \delta \dot{\mathbf{u}} d \Omega+\int_{\Gamma_{N}} \mathbf{h} \delta \dot{\mathbf{u}} d \Gamma_{N}
$$

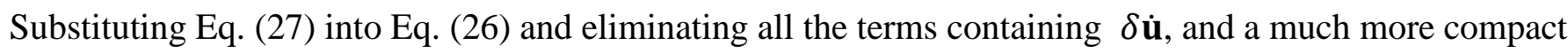
form of Eq. (26) can be obtained, as:

$$
\int_{\Omega} \boldsymbol{\varepsilon}(\dot{\mathbf{u}})^{T} \mathbf{D}(\mathcal{X}) \boldsymbol{\varepsilon}(\delta \mathbf{u}) d \Omega=-\int_{\Omega} \boldsymbol{\varepsilon}(\mathbf{u})^{T} \frac{\partial \mathbf{D}(\mathcal{X})}{\partial \mathcal{X}} \boldsymbol{\varepsilon}(\delta \mathbf{u}) d \Omega
$$

It is known that the static compliance problems are self-adjoint [1]. Eq. (28) can be expanded as a form:

$$
\int_{\Omega} \boldsymbol{\varepsilon}(\dot{\mathbf{u}})^{T} \mathbf{D}(\mathcal{X}) \boldsymbol{\varepsilon}(\mathbf{u}) d \Omega=-\int_{\Omega} \boldsymbol{\varepsilon}(\mathbf{u})^{T} \frac{\partial \mathbf{D}(\mathcal{X})}{\partial \mathcal{X}} \boldsymbol{\varepsilon}(\mathbf{u}) d \Omega
$$

Substituting Eq. (29) into Eq. (25). The first-order derivative of the objective function with respect to the DDF is gained, explicitly by:

$$
\frac{\partial J}{\partial \chi}=-\frac{1}{2} \int_{\Omega} \boldsymbol{\varepsilon}(\mathbf{u})^{T} \frac{\partial \mathbf{D}(\mathcal{X})}{\partial \mathcal{X}} \boldsymbol{\varepsilon}(\mathbf{u}) d \Omega
$$

It can be easily seen that the final form of the sensitivity of the optimized objective function depends on the derivative of the elastic tensor with respect to the DDF. In Eq. (22), material elastic tensor is an exponential function of the DDF, and the derivative of the objective with respect to the DDF is expressed as:

$$
\frac{\partial J}{\partial \chi}=-\frac{1}{2} \int_{\Omega} \boldsymbol{\varepsilon}(\mathbf{u})^{T} \gamma \chi^{\gamma-1} \mathbf{D}_{0} \boldsymbol{\varepsilon}(\mathbf{u}) d \Omega
$$

Similarly, the first-order derivative of the volume constraint with respect to the DDF is given as:

$$
\frac{\partial G}{\partial \chi}=\frac{1}{|\Omega|} \int_{\Omega} v_{0} d \Omega
$$


In Section 3, the DDF is constructed by the NURBS basis functions with the smoothed nodal densities, and the smoothed nodal densities are developed by the Shepard function. The first-order sensitivity of the DDF with respect to the smoothed nodal densities of control points are derived by:

$$
\frac{\partial \mathcal{X}(\xi, \eta, \zeta)}{\partial \mathcal{G}\left(\rho_{i, j, k}\right)}=R_{i, j, k}^{p, q, r}(\xi, \eta, \zeta)
$$

where $R_{i, j, k}^{p, q, r}(\xi, \eta, \zeta)$ denote the NURBS basis function at the computational point $(\xi, \eta, \zeta)$. In Eq. (14), the derivatives of the smoothed nodal densities with respect to nodal densities can be expressed by:

$$
\frac{\partial \mathcal{G}\left(\rho_{i, j, k}\right)}{\partial \rho_{i, j, k}}=\psi\left(\rho_{i, j, k}\right)
$$

where $\psi\left(\rho_{i, j, k}\right)$ is the value of the Shepard function at the current control point $(i, j, k)$. It is important to note that the above computational point $(\xi, \eta, \zeta)$ is different from the control point $(i, j, k)$. The control points may be not on the domain and only work as control coefficients to construct the DDF. However, the computational points are located at the design domain. In the current formulation, the computational points are the Gauss quadrature points. The first-order derivatives of the objective and constraint functions with respect to nodal densities are derived based on the chain rule, and the final detailed form of the first-order derivatives is explicitly described by:

$$
\left\{\begin{array}{l}
\frac{\partial J}{\partial \rho_{i, j, k}}=-\frac{1}{2} \int_{\Omega} \boldsymbol{\varepsilon}(\mathbf{u})^{T} \gamma(\chi(\xi, \eta, \zeta))^{\gamma-1} R_{i, j, k}^{p, q, r}(\xi, \eta, \zeta) \psi\left(\rho_{i, j, k}\right) \mathbf{D}_{0} \boldsymbol{\varepsilon}(\mathbf{u}) d \Omega \\
\frac{\partial G}{\partial \rho_{i, j, k}}=\frac{1}{|\Omega|} \int_{\Omega} R_{i, j, k}^{p, q, r}(\xi, \eta, \zeta) \psi\left(\rho_{i, j, k}\right) v_{0} d \Omega
\end{array}\right.
$$

It can be seen that the first-order derivatives of the objective function are negative due to the nonnegativity of the NURBS basis functions and the Shepard function. Meanwhile, the sensitivity analysis in Eq. (35) is cost-effective, due to the fact that the Shepard function and NURBS basis functions are only dependent on the spatial locations of the control points and Gauss quadrature points, respectively. They can be pre-stored during the optimization for continuum structures.

\section{Numerical implementations}

The OC method [57] is applied to solve the formulation due to its superior characteristic for the optimization problems with a large number of design variables and only a few constraints. An efficient heuristic updating scheme is given and the detailed formulation can refer to $[16,21,23]$, as: 


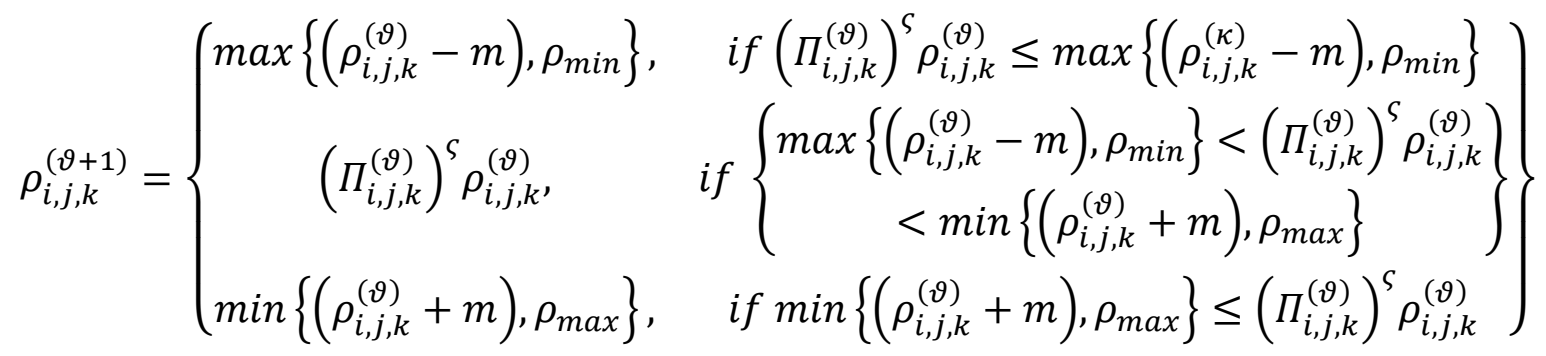

where $m$ and $\varsigma$ are the move limit and the damping factor, respectively. $\Pi_{i, j, k}^{(\vartheta)}$ is the updating factor for the $(i, j, k)$ design variable at the $\vartheta^{\text {th }}$ iterative step.

$$
\Pi_{i, j, k}^{(\vartheta)}=-\frac{\partial J}{\partial \rho_{i, j, k}} / \max \left(\mu, \Lambda^{(\vartheta)} \frac{\partial G}{\partial \rho_{i, j, k}}\right)
$$

where $\mu$ is a very small positive constant to avoid the zero in the denominator. $\Lambda^{(\vartheta)}$ is the Lagrange multiplier at the $\vartheta^{\text {th }}$ iteration step, which is updated by a bi-sectioning algorithm [1].

The flowchart of the current developed IGATO framework is displayed in Fig. 3. The main parts are given within red boxes, namely: (1) The geometrical model constructed by NURBS; (2) The development of the numerical analysis model (the IGA mesh); (3) The improvement of the smoothness of the nodal densities by the Shepard function; (4) The construction of the DDF using NURBS for 2D and 3D; (5) The evaluation of the unknown structural responses by the NURBS-based IGA; (6) The evolving of the DDF.

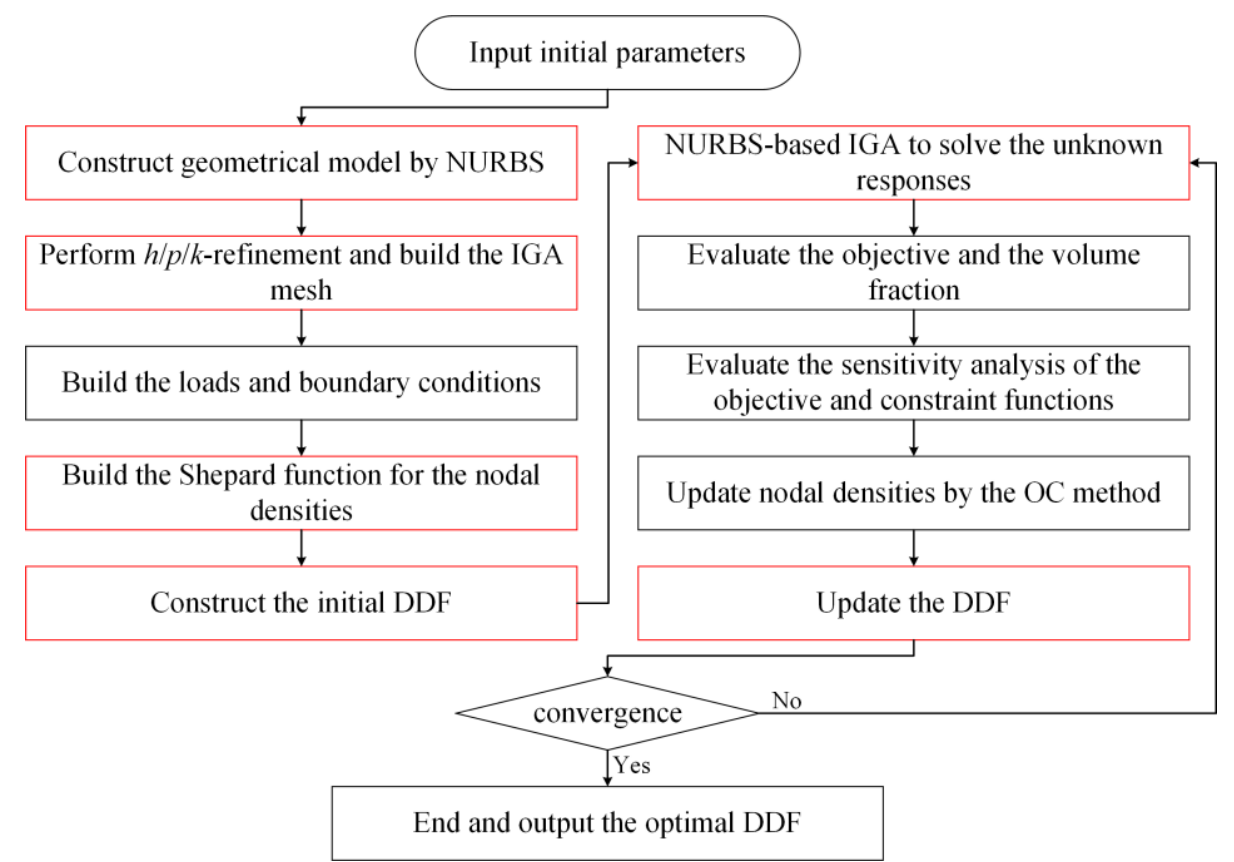

Fig. 3. The flowchart of the ITO method

\section{Numerical Examples}

In this section, several numerical examples in 2D and 3D are provided to demonstrate the effectiveness and efficiency of the developed ITO method. The classic cantilever beam is firstly studied to show the benefits 
of the refined DDF during the optimization. Then, a quarter annulus with the curved design domain, and a complex structure meshed by multiple patches in IGA, as well as a 3D Michell structure and 3D Bridgetype structure, are discussed to present the effectiveness of the ITO method. In all examples, the Young's moduli for solid materials are defined as 1 . The Poisson's ratio is set to be 0.3 . The imposed point load magnitude is defined as 1 . The Gauss quadrature points with $3 \times 3(2 \mathrm{D})$ or $3 \times 3 \times 3(3 \mathrm{D})$ are chosen in each IGA element. Meanwhile, it is noted that no extra filtering schemes are contained. The penalty factor $\gamma$ is defined as 3 . The terminal criterion is that the $L_{\infty}$ norm of the difference of the nodal densities between two consecutive iterations is less than $1 \%$ or the maximum 150 iteration steps are reached.

\subsection{Cantilever beam}

The structural design domain of the cantilever beam with the loads and boundary conditions is defined in Fig. $4(a)$, and two indices $L$ and $H$ are defined as 10 and 5, respectively. The NURBS surface to model the cantilever beam with the control net of points is displayed in Fig. $4(b)$ and the IGA mesh to discrete the design domain with Gauss quadrature points is shown in Fig. $4(c)$. The related modeling details are attached below Fig. 4. The allowable material volume fraction $V_{0}$ is set as $30 \%$. As shown in Fig. $5(a)$, an initial set of the nodal densities is provided, and all values are equal to 1. The corresponding densities at Gauss quadrature Points are presented in Fig. $5(b)$, and the DDF of the cantilever beam is displayed in Fig. $5(c)$.

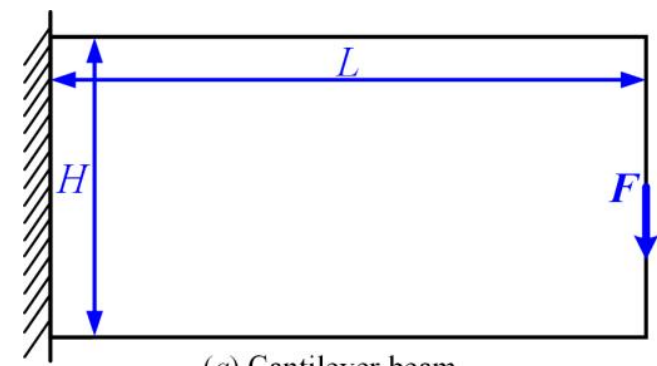

(a) Cantilever beam

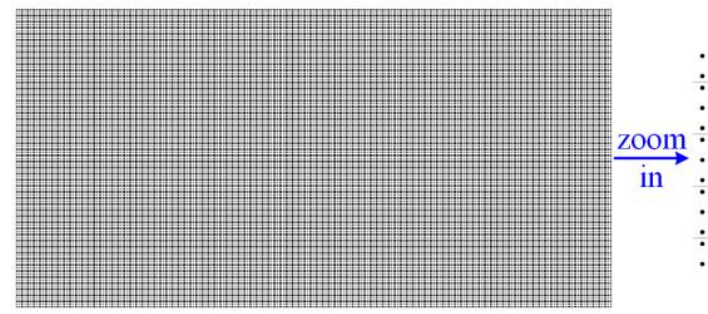

(c) IGA mesh with Gauss points

Fig. 4. Cantilever beam: IGA elements $100 \times 50 ; \Xi=\{0,0,0,0,0.01, \cdots, 0.99,1,1,1,1\}, \mathcal{H}=$ $\{0,0,0,0,0.02, \cdots, 0.98,1,1,1,1\} ; n=103, m=l=53 ; p=q=3$.

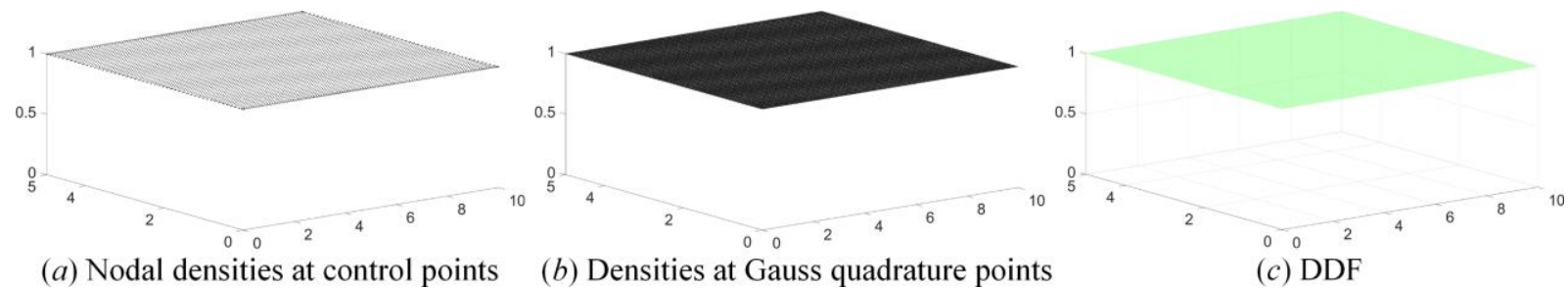

Fig. 5. The initial design of cantilever beam 
The optimized results are displayed in Fig. 6, including the optimized distribution of nodal densities at the control points in Fig. 6 (a), densities at Gauss quadrature points in Fig. 6 (b) and the DDF in Fig. 6 (c). As we can see, the optimized response surface of the DDF is characterized with the sufficient smoothness and continuity, which will be beneficial to represent the structural topology. As already pointed out in Section 3, it mainly originates from the Shepard function and NURBS basis functions. The former can guarantee the overall smoothness of nodal densities at control points. The latter makes sure the high-order continuity of the DDF, considerably depended on the orders of NURBS basis functions. Meanwhile, the intermediate results of the DDF during the optimization from iterations 1 to 85 are also shown in Fig. 7. As we can see, the updating of the DDF in the optimization is featured with the high stability.

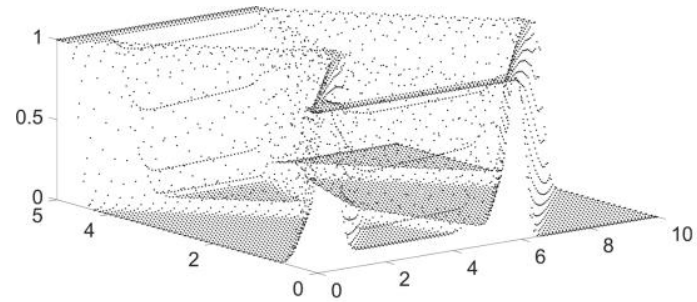

(a) Nodal densities at control points

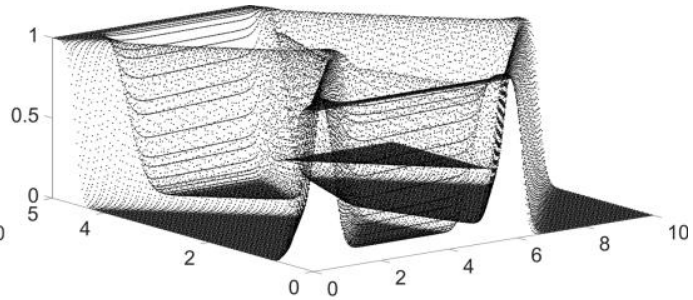

(b) Densities at Gauss quadrature points

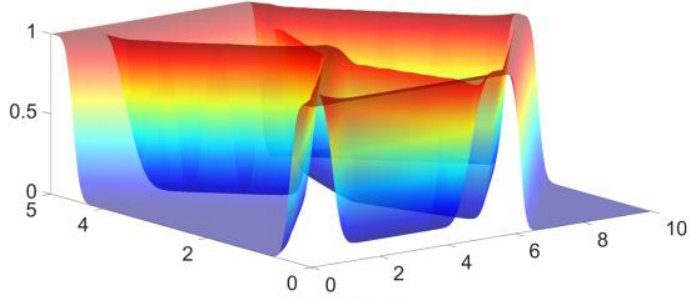

(c) DDF

Fig. 6. The optimized results of cantilever beam

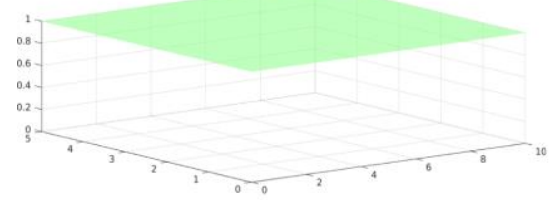

(a) Iteration 1

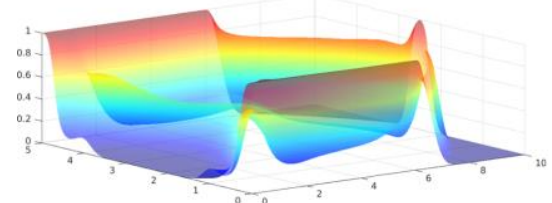

(d) Iteration 12

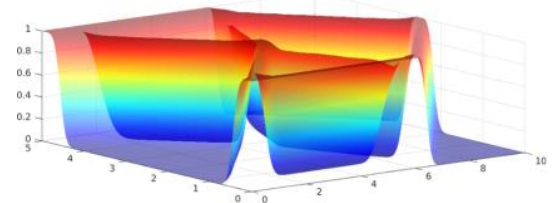

(g) Iteration 37

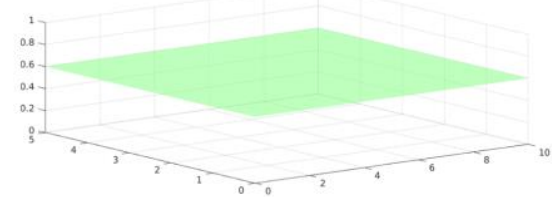

(b) Iteration 3

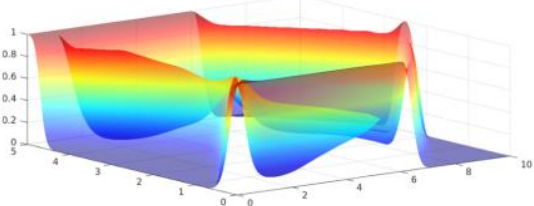

(e) Iteration 15

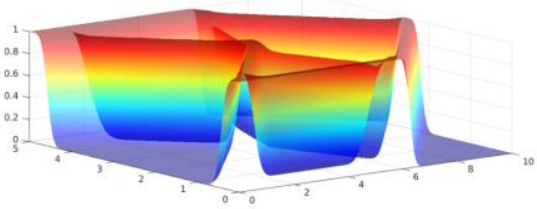

(h) Iteration 55

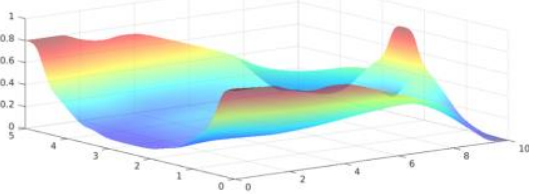

(c) Iteration 6

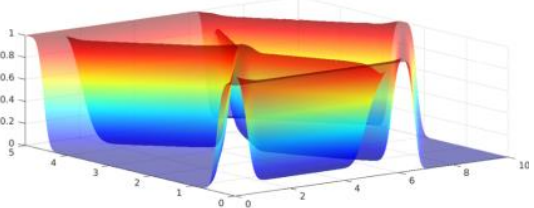

(f) Iteration 25

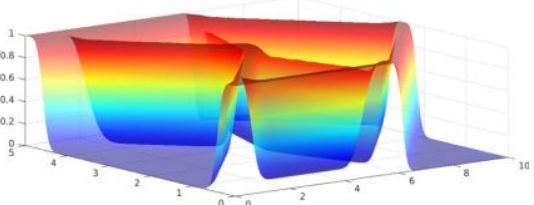

(i) Iteration 85

Fig. 7. The intermediate DDFs

As displayed in Fig. 6, the optimized densities in the DDF mostly approach the lower or upper bounds, namely 0 or 1 . In order to achieve an appropriate architecture of the cantilever beam, a heuristic criterion 
is introduced to define the structural topology using the optimized DDF. The corresponding mathematical model is expressed in Eq. (38), as:

$$
\left\{\begin{array}{cc}
0 \leq \mathcal{X}(\xi, \eta)<x_{c} & \text { void } \\
\mathcal{x}(\xi, \eta)=\chi_{c} & \text { boundary } \\
x_{c}<\mathcal{X}(\xi, \eta) \leq 1 & \text { solid }
\end{array}\right.
$$

where $X_{c}$ is a constant. Hence, structural boundaries of the optimized topology are expressed by the isocontour of the response surface expressed by the DDF. The densities smaller than $\chi_{c}$ of the DDF denote voids in the optimized topology and the DDF with the densities larger than $\chi_{c}$ describe the solids. It can be easily seen that the heuristic scheme to define the structural topology using the DDF is analogous to the LSM [6-8]. However, it is noted that the proposed ITO method for the optimization of continuum structures is not developed in a framework of the Hamilton-Jacobi partial differential equation. The mechanism in Eq. (38) can be just viewed as a post-processing scheme to define the topology. The core of the developed ITO method is the optimization of the sufficiently smooth and continuous DDF.

In this work, $X_{c}=0.5$ is a relatively suitable value to define the topology using the DDF, owing to the optimized densities of the DDF are mostly distributed nearly 0 or 1 . The final topology of the cantilever beam is provided in the second column of Table 1, and densities at Gauss quadrature points with the values larger than 0.5 in a 2D view are also given in Table 1 . It can be easily seen that the $2 \mathrm{D}$ view with $\mathcal{X} \geq 0.5$ is analogous to the topology. A minor difference is that the topology is featured with the sufficiently smooth boundaries and clear interfaces between the solids and voids. Meanwhile, the optimized objective function and the total iterative step are also given, namely $O b j=129.88$ and $N u m=85$. A limited number of the iterations to arrive at the convergent condition displays the high efficiency of the ITO method. Additionally, the well-known numerical artifacts, such as the checkerboards, zig-zag or wavy boundaries, islanding or layering structures, can be effectively eliminated in the optimized topology.

Table 1. The optimized results of the cantilever beam

The 2D-view DGQP

Finally, the iterative curves for the objective function and volume fraction are displayed in Fig. 8, with the red curve to represent the evolving of the objective function and the blue curve to show the variation of the 
volume fraction. It can be easily seen that the convergent curves are characterized with a clear, smooth and fast convergence, which shows the effectiveness and efficiency of the ITO method. It should be noted that iterative curve of the volume fraction in Fig. 8 denotes the evolving of the DDF, rather than the topology. The intermediate results of the topology are also provided in Fig. 8. After the optimization, we re-calculate the volume fraction in terms of the topology, where the densities should be re-computed by modifying the DDF, that is $x \leftarrow 1$ (if $x \geq 0.5$ ) and $x \leftarrow 0$ (if $x<0.5$ ). The volume fraction of the topology is equal to $30.25 \%$, which is slightly different from the prescribed maximum volume fraction $V_{0}=30 \%$. Hence, the constant $X_{c}=0.5$ is an appropriate value in the definition of the topology using the DDF.

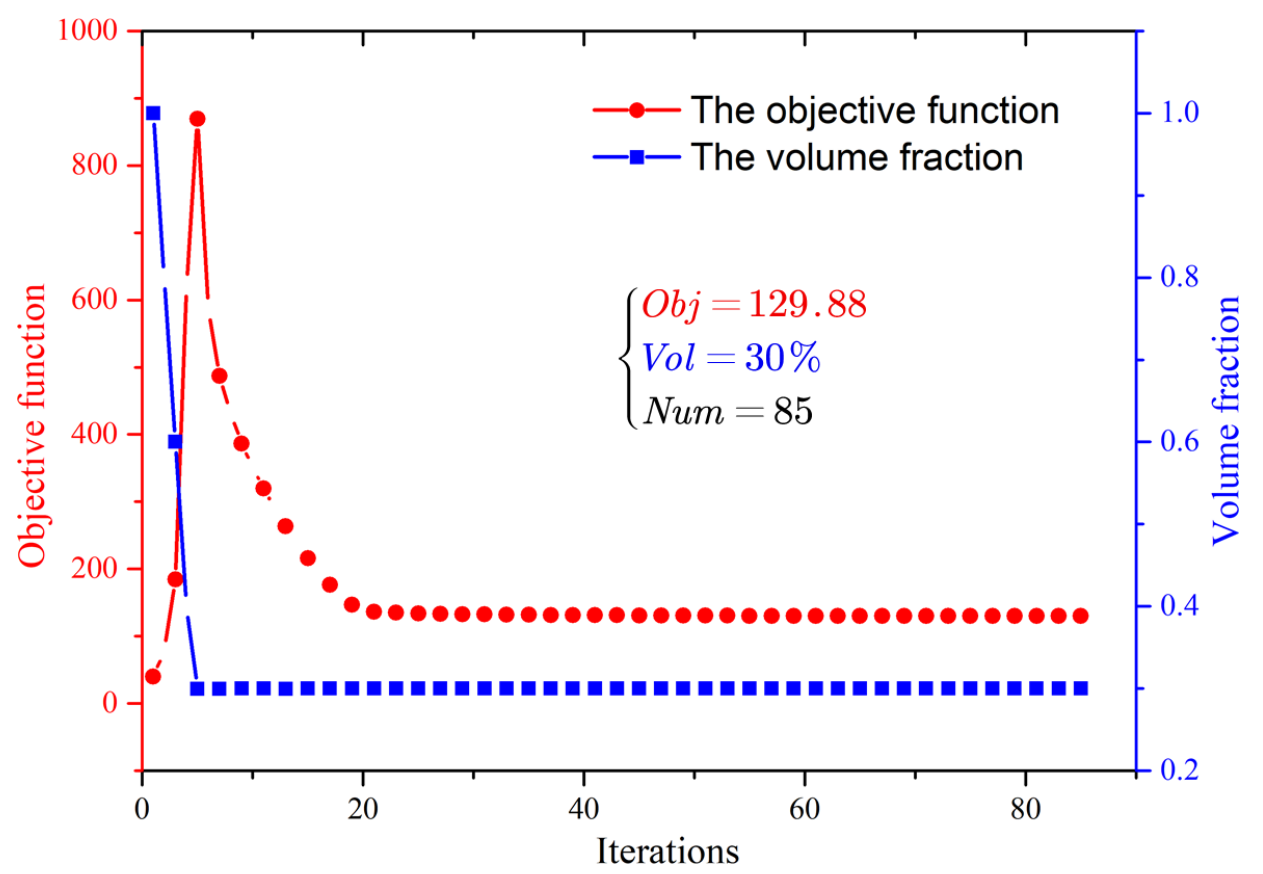

Fig. 8. Convergent histories

\subsubsection{Discussions of mesh dependency}

In this subsection, we provide the extensive discussions of the influence of the IGA mesh. The knot vectors in three cases are listed in Table 2, and the corresponding IGA meshes have $80 \times 40,120 \times 60$ and $140 \times 70$ elements in cases 1, 2 and 3, respectively. The numbers of control points are $83 \times 43,123 \times 63$ and $143 \times 73$, respectively. The maximum material volume fraction is still set as $30 \%$.

Table 2. Knot vectors in three cases

\begin{tabular}{cc}
\hline Case & Knot vectors \\
\hline 1 & $\Xi=\{0,0,0,0,0.0125, \cdots, 0.9875,1,1,1,1\}, \mathcal{H}=\{0,0,0,0,0.0250, \cdots, 0.9750,1,1,1,1\}$ \\
\hline 2 & $\Xi=\{0,0,0,0,0.0083, \cdots, 0.9917,1,1,1,1\}, \mathcal{H}=\{0,0,0,0,0.0167, \cdots, 0.9833,1,1,1,1\}$ \\
\hline 3 & $\Xi=\{0,0,0,0,0.0071, \cdots, 0.9929,1,1,1,1\}, \mathcal{H}=\{0,0,0,0,0.0143, \cdots, 0.9857,1,1,1,1\}$ \\
\hline
\end{tabular}

The initial values of nodal densities at control points are still defined to be 1 . The optimized results of three cases are presented in Fig. 9, including densities at Gauss quadrature points, the 2D view of densities with the values larger than 0.5 , the DDF and the topology. The corresponding numerical results in three cases, 
consisting of the optimized objective function, the total iterations, the volume fraction of the topology are listed in Table 3, respectively. As we can see, the optimized DDFs and topologies in three cases are mostly identical, which demonstrates the ability of the ITO method to eliminate the mesh dependency. The main cause is that the NURBS for the representation of the DDF has the intrinsic filter [46]. As discussed in [46], the filter scale is dependent on the orders of NURBS basis functions and the knot spans in all parametric directions. Moreover, the topology in Case 3 with the finest IGA mesh has the best stiffness performance, and its volume fraction is also closest to the prescribed maximum material consumption $30 \%$, while more iterations are required to meet the convergent criterion owing to the increasing of design variables. In terms of choosing the IGA mesh in the optimization, it is also a trade-off between the computational cost and numerical precision. Generally speaking, a mesh with $100 \times 50$ is a great choice.

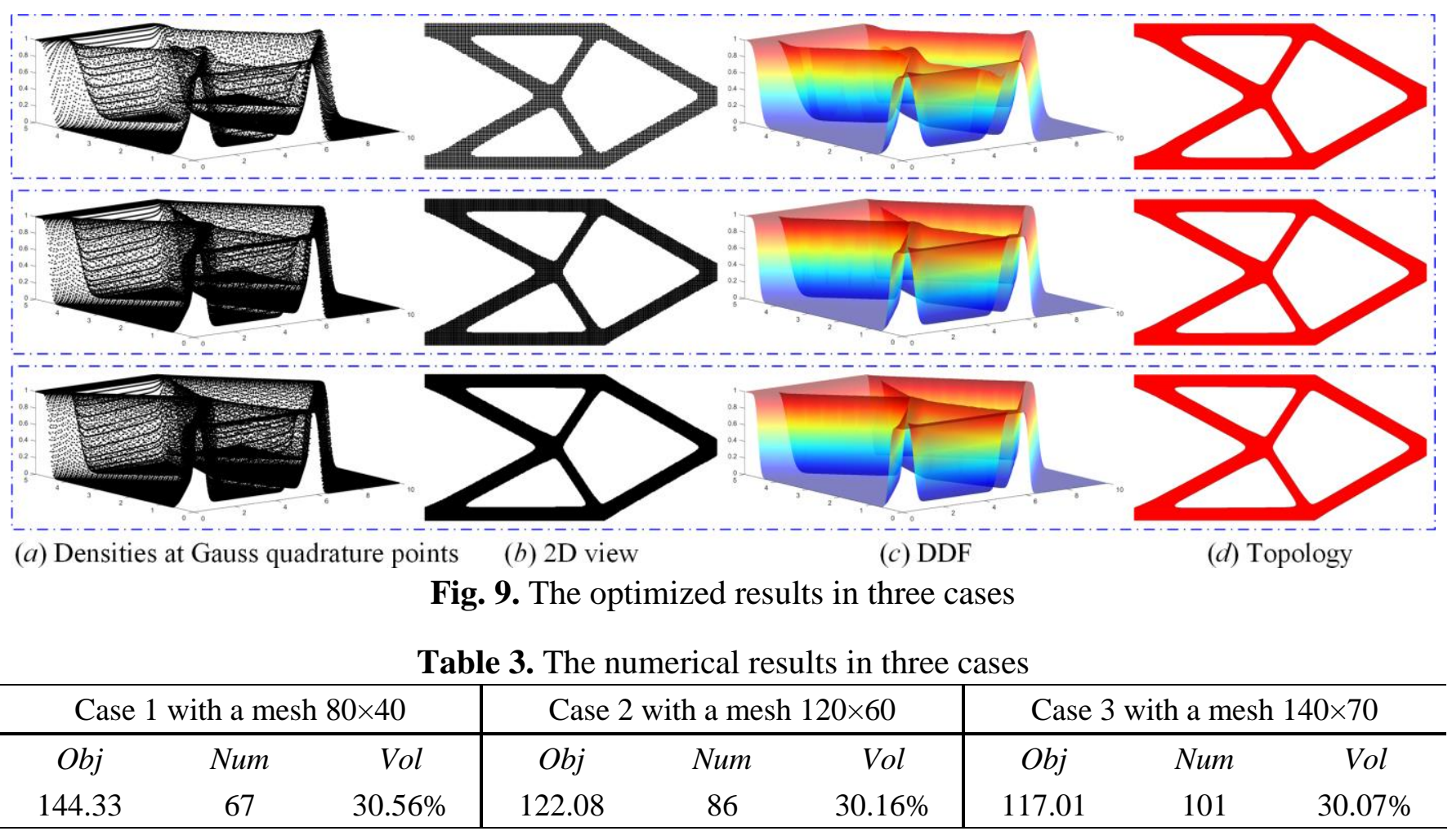

\subsubsection{Discussions of the Shepard function}

In this subsection, two numerical cases are performed to address the influence of the Shepard function on the optimization, where the construction of the DDF does not employ the Shepard function to improve the smoothness of nodal densities. Two cases have different orders of NURBS basis functions, namely the 1order basis functions in Case 1 and 3-order in Case 2. Other parameters are consistent with the first example, and the initial design of the cantilever beam in Fig. $\mathbf{5}$ is utilized in two cases.

The optimized results of two cases are provided in Table 4, including the DDF, the topology, the objective function, the total steps, and the volume fraction of the topology. It can be seen that the optimized results 
in Case 1 are featured with "islanding" or "layering" structures, "zig-zag" or wavy structural boundaries. However, the numerical issue of the "islanding" or "layering" structures is removed in the final topology of Case 2. In order to show the influence of the Shepard function and the order of basis functions on the "islanding" and "layering" numerical artifact in detail, the intermediate topologies in Cases 1 and 2 are presented in Fig. 10, respectively. As we can see, the "islanding" and "layering" structures are easily occurred during the optimization in Case 1, as shown in Fig. $10(a c),(a d)(a e)$ and $(a f)$. The NURBS filter is inactive in the elimination of the numerical issue, if the order is equal to 1 [46]. Elevating the order of the NURBS basis functions can increase the filter scale. As shown in Fig. $10(b d)$, the formed "islanding" and "layering" structures in Case 2 can be removed in the final topology.

Table 4. The optimized results in two cases

\begin{tabular}{llllll}
\hline & Case 1 with 1-order & Obj Num & Nol \\
\hline DDF & Topology & None $30.88 \%$ \\
\hline
\end{tabular}

\section{Case 2 with 3-order}

(1) 30.49\%

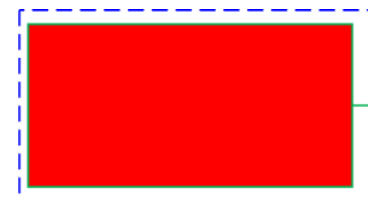

(a) Iteration 1

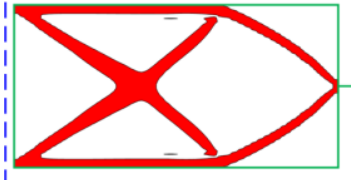

(c) Iteration 15

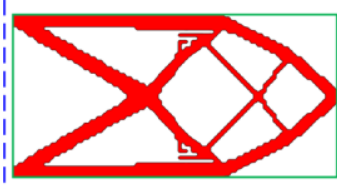

(e) Iteration 53

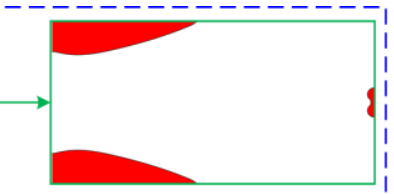

(b) Iteration 5

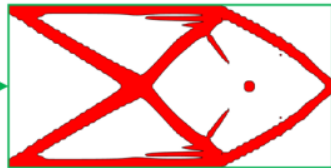

(d) Iteration 20

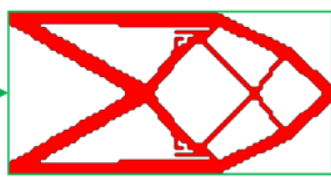

(f) Iteration 151

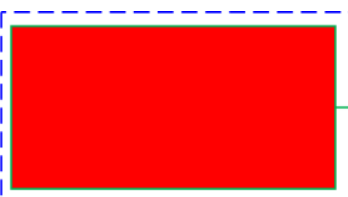

(a) Iteration 1

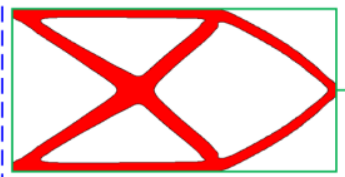

(c) Iteration 15

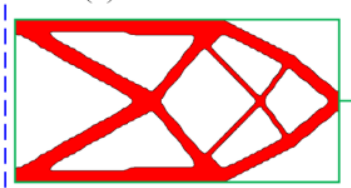

(e) Iteration 63

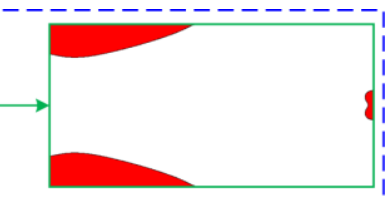

(b) Iteration 5

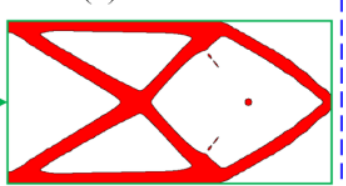

(d) Iteration 20

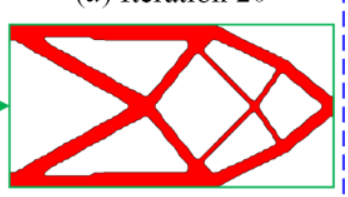

(f) Iteration 151

(a) Case 1

Fig. 10. Intermediate topologies

As shown in Table 4, the "zig-zag" or wavy structural boundaries are existed in the optimized topologies of Cases 1 and 2, which introduce some difficulties for the latter manufacturing. Similar to the results in 
[43] where densities at control points are applied to represent the structural topology, the wavy and blurry structural boundaries are also occurred, due to a fact that the overall smoothness of nodal densities is not improved. Meanwhile, the optimizations in two cases are not stable, and the final change of nodal densities between consecutive steps cannot satisfy the defined criterion. Hence, we can confirm that it is imperative to consider the Shepard function in the construction of the DDF, not only ensure the sufficient smoothness and continuity, but also improve the stability of the optimization.

\subsubsection{Discussions of the initial design}

In this subsection, the influence of the initial design on the optimization will be investigated in three cases, where three different initial designs of nodal densities are employed, as shown in Fig. 11. As we can see, nodal densities are homogenously distributed and equal to 0.5 and 0.3 , respectively. In the initial design 3 , the definition of nodal densities is not uniformly configured in the structural design domain, with a part of nodal densities equal to 0. Other design parameters keep consistent with the first example, shown in Fig. 4. The maximum material consumptions in three cases are equal to $30 \%$.

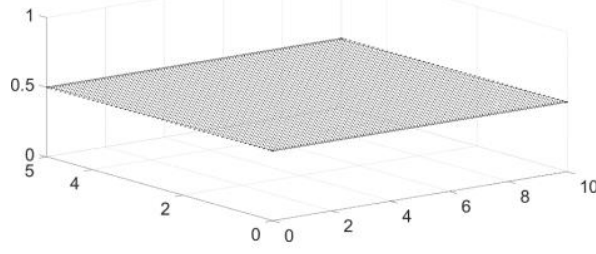

(a) Initial design 1

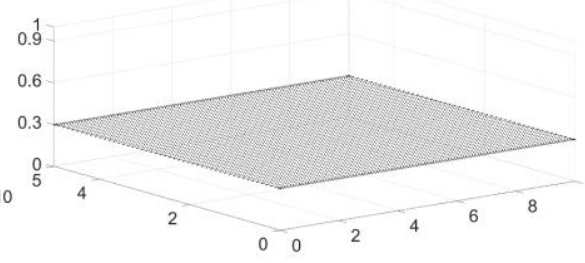

(b) Initial design 2

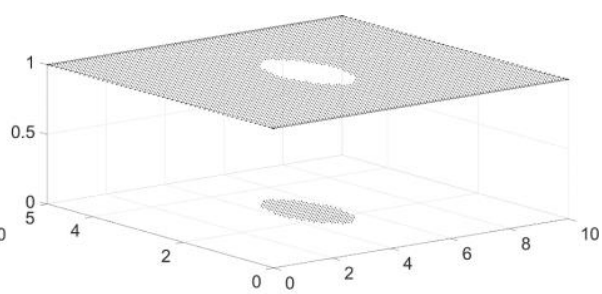

(c) Initial design 3

Fig. 11. Nodal densities at control points in three cases

The optimized designs in three cases are given in Fig. 12, including the optimized densities at Gauss points, the DDF and the topology. It can be easily seen that the optimized topologies are mostly identical in three cases, and only the topology in case 3 has the minor difference. The related numerical results of three cases are listed in Table 5, containing the optimized objective function, the total convergent steps and the volume fraction of the topology. As we can see, the objective functions in three cases are mostly identical, and only Case 3 has a larger structural mean compliance, namely 131.32. We can confirm that the initial design of nodal densities in a homogenous distribution has a negligible effect on the latter optimization, as shown in Table 1 and Fig. 12. Moreover, the homogenous nodal densities are beneficial to the latter optimization of structures, and the non-uniform distributed nodal densities might lead to a local optimum.

Table 5. The numerical results in three cases

\begin{tabular}{|c|c|c|c|c|c|c|c|c|}
\hline \multicolumn{3}{|c|}{ Case 1 with the initial design 1} & \multicolumn{3}{|c|}{ Case 2 with the initial design 2} & \multicolumn{3}{|c|}{ Case 3 with the initial design 3} \\
\hline Obj & Num & Vol & Obj & Num & Vol & Obj & Num & Vol \\
\hline 130.15 & 72 & $30.12 \%$ & 130.08 & 71 & $30.12 \%$ & 131.32 & 82 & $30.54 \%$ \\
\hline
\end{tabular}




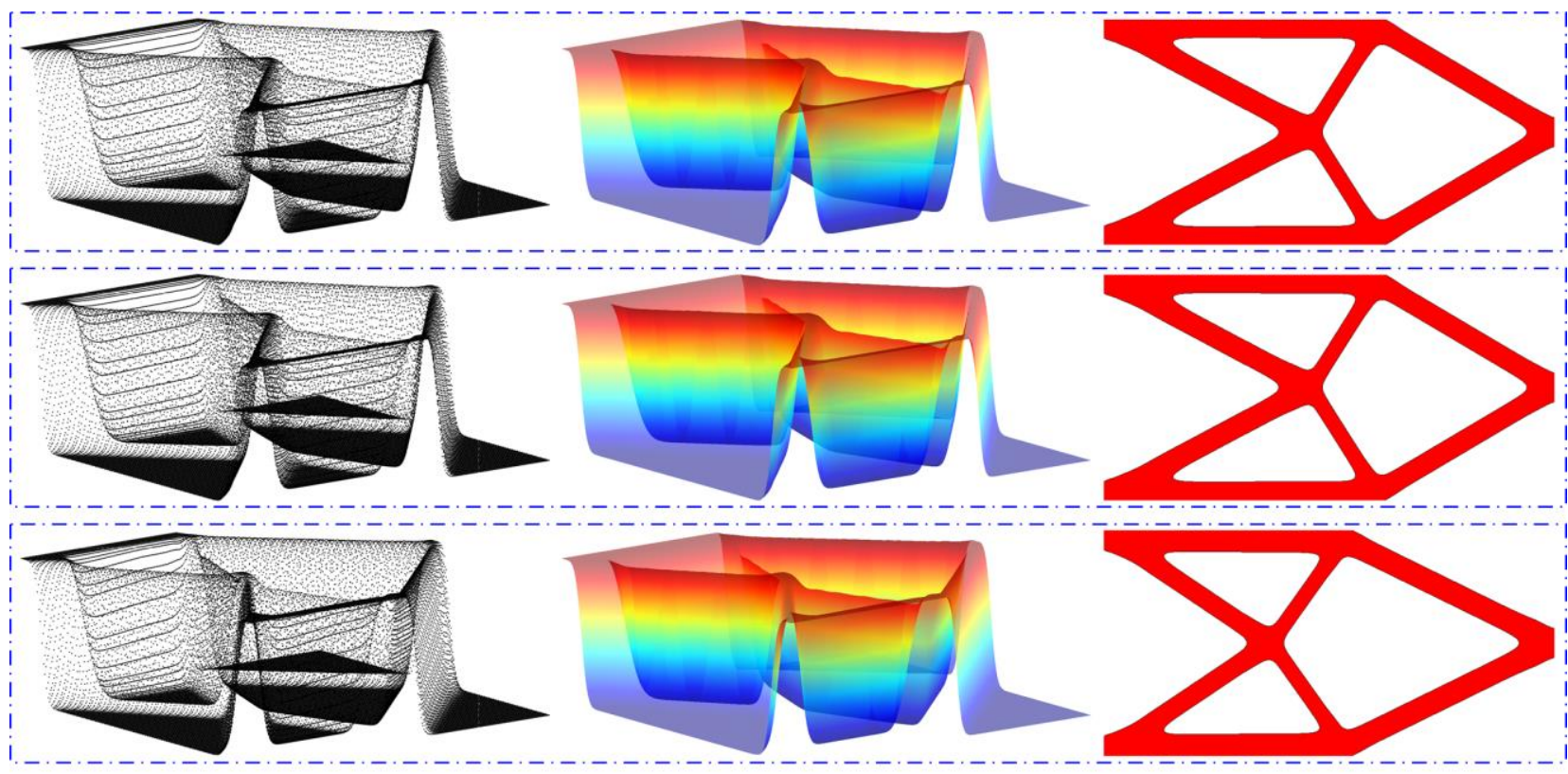

(a) Densities at Gauss quadrature points (b) DDF (c) Topology

Fig. 12. The optimized designs in three cases

\subsection{Quarter annulus}

In this subsection, a quarter annulus with the loads and boundary conditions is defined Fig. 13 (a), and two indices $r$ and $R$ are set as 5 and 10, respectively. The NURBS surface with control points and the IGA mesh with Gauss quadrature points are shown in Fig. $13(b)$ and $(c)$, respectively. The maximum material volume fraction is set as $40 \%$. The initial design of the quarter annulus is shown in Fig. 14.

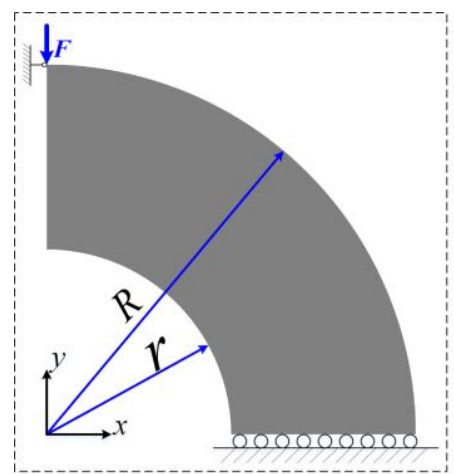

(a) Quarter annulus

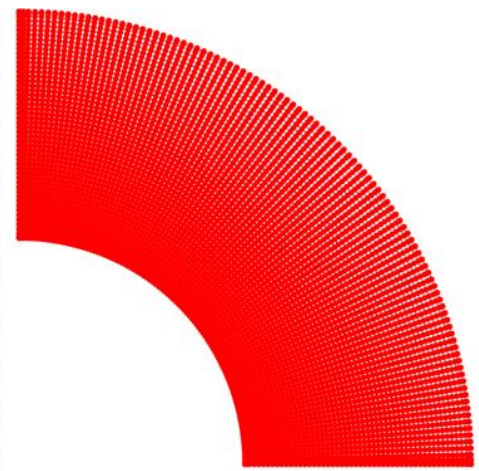

(b) NURBS surface

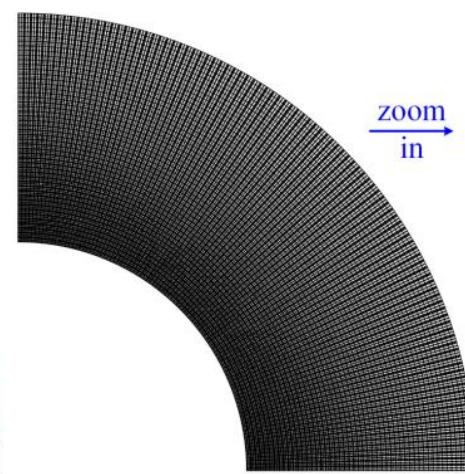

(c) IGA mesh with Gauss points

Fig. 13. Quarter annulus: IGA elements $100 \times 50 ; \Xi=\{0,0,0,0,0.01, \cdots, 0.99,1,1,1,1\}, \mathcal{H}=$ $\{0,0,0,0,0.02, \cdots, 0.98,1,1,1,1\} ; n=103, m=l=53 ; p=q=3$.

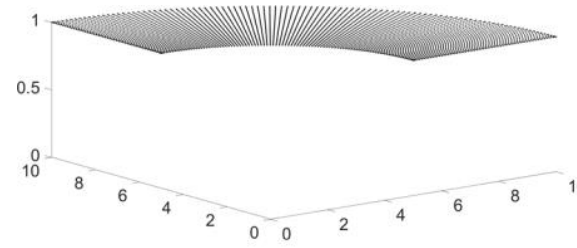

(a) Nodal densities at control points

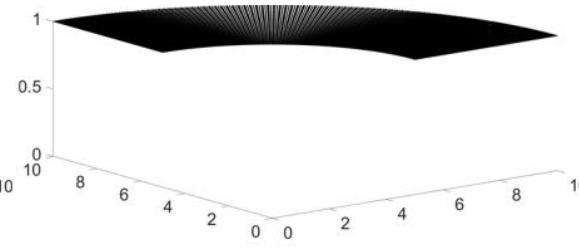

(b) Densities at Gauss quadrature points

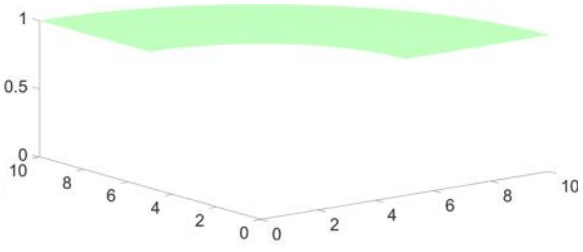

(c) $\mathrm{DDF}$

Fig. 14. The initial design of quarter annulus

The optimized designs, including densities at Gauss quadrature points, the 2D view of densities larger than 0.5, the DDF and topology, are shown in Fig. 15. Similar to Section 6.1, the optimized topology is featured with the smooth structural boundaries and distinct interfaces between the solids and voids. Meanwhile, the 
DDF is also featured with the sufficient smoothness and continuity, and the intermediate DDFs during the optimization are also shown in Fig. 16. As we can see, the evolving of the DDF is featured with the high stability and can quickly reach the defined convergent criterion, which can demonstrate the effectiveness and efficiency of the proposed ITO method.

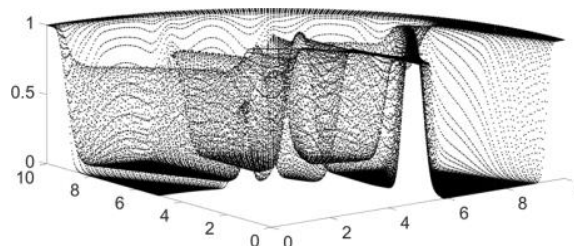

(a) Densities at Gauss quadrature points

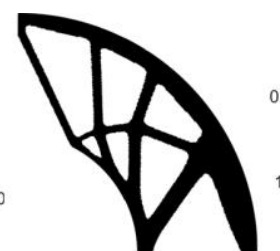

(b) $2 \mathrm{D}$ view

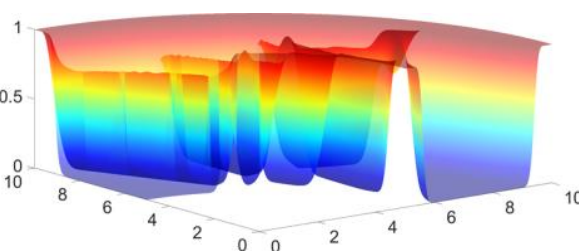

(c) DDF

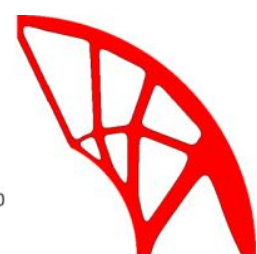

(d) Topology

Fig. 15. The optimized results of quarter annulus

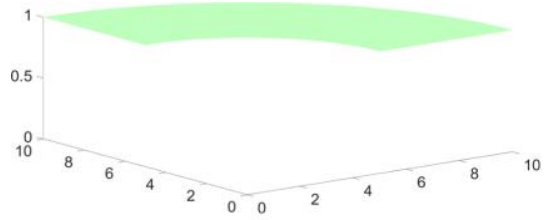

(a) Iteration 1

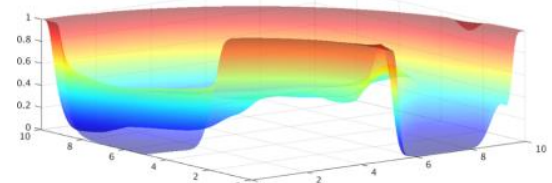

(d) Iteration 10

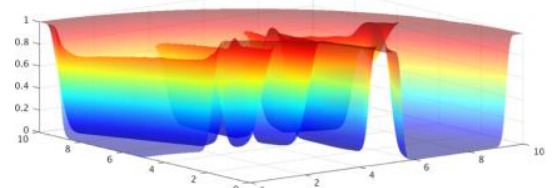

(g) Iteration 55

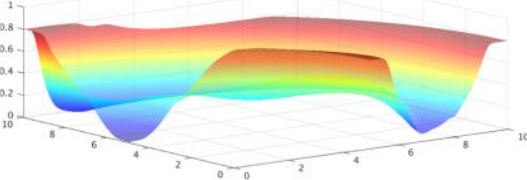

(b) Iteration 5

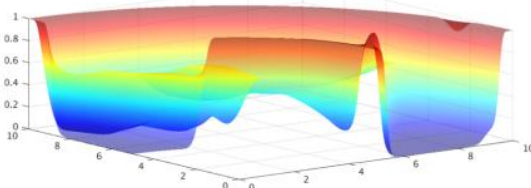

(e) Iteration 13

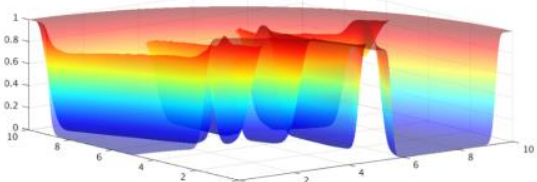

(h) Iteration 74

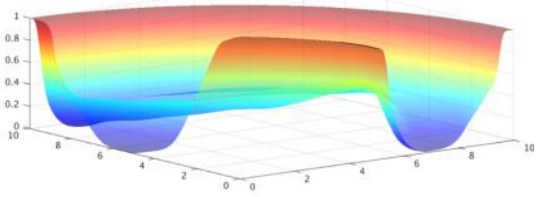

(c) Iteration 7

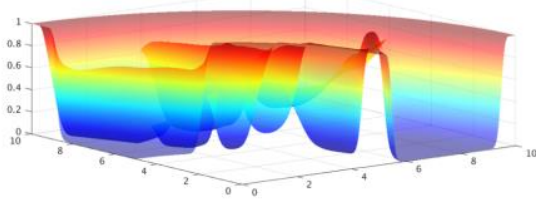

(f) Iteration 21

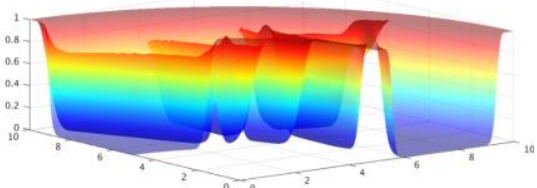

(i) Iteration 97

Fig. 16. The intermediate DDFs

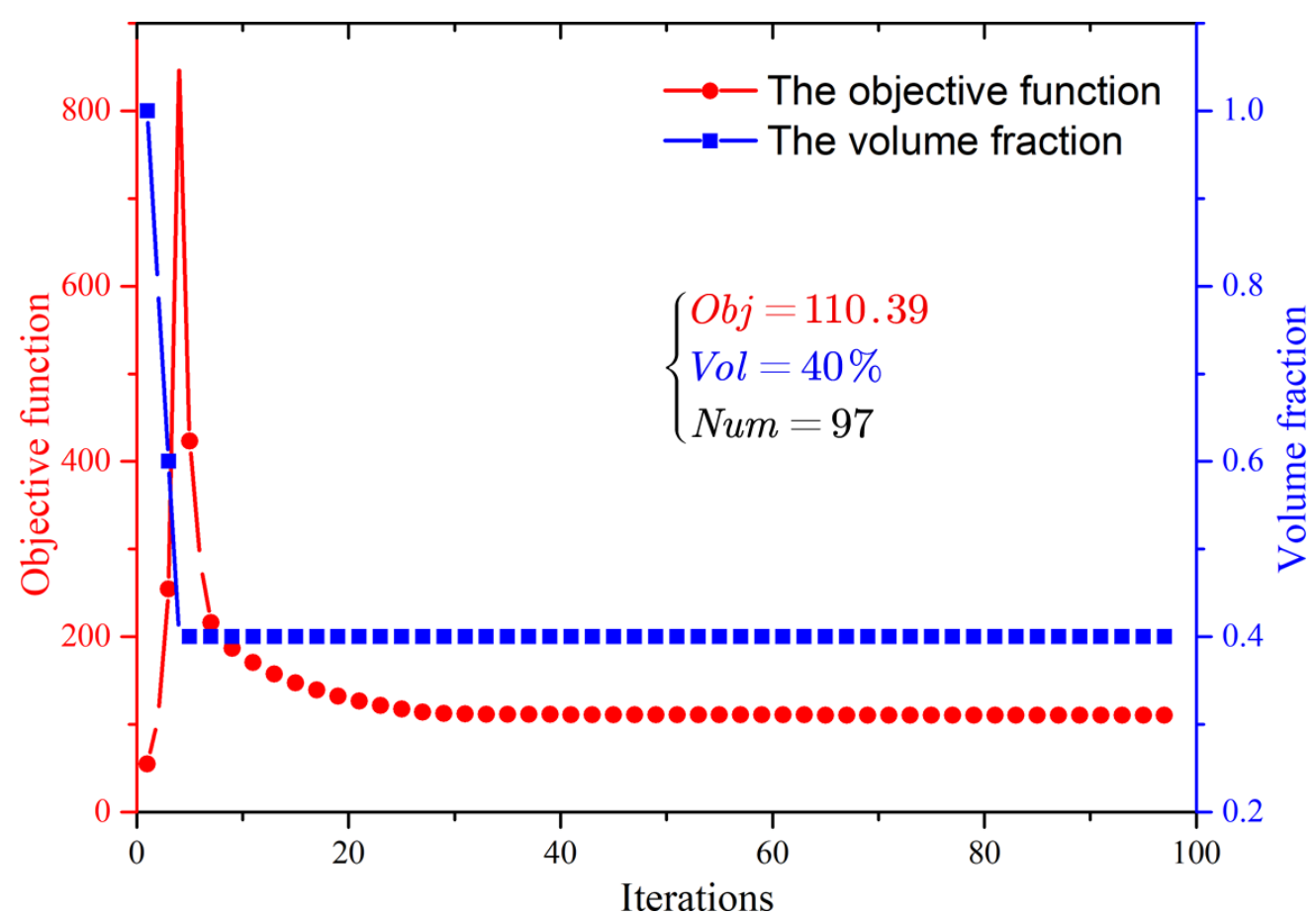

Fig. 17. Convergent histories 
Meanwhile, the iterative histories of the objective function and volume fraction of the DDF are also shown in Fig. 17. It can be easily seen that the volume fraction of the DDF can quickly arrives at the prescribed maximum material consumption. Then, the DDF is gradually advanced to improve the structural stiffness performance. The major structural features are formed in the former iterations (nearly 30 steps) and more iterations are spent to slightly adjust the geometric details until the optimal structure is obtained. Moreover, the intermediate topologies are also attached with the display of the iterative curves, shown in Fig. 17. The volume fraction of the final topology is also re-calculated, equal to $40.41 \%$. Hence, we can confirm that the heuristic scheme to define the structural topology using the DDF is relatively applicable.

\subsubsection{Discussions of the order of NURBS basis functions}

This subsection aims to address the influence of the order of NURBS basis functions on the optimization of the curved structures. The order of the NURBS basis functions is defined in three cases, namely 2,3 and 4. The corresponding knot vectors are defined in Table 6. The maximum material consumptions in three cases are all defined as $40 \%$. The initial values of nodal densities in three cases are all equal to 1 .

Table 6. Knot vectors in three cases

\begin{tabular}{cc}
\hline Case & Knot vectors \\
\hline 1 & $\Xi=\{0,0,0,0.01, \cdots, 0.99,1,1,1\}, \mathcal{H}=\{0,0,0,0.02, \cdots, 0.98,1,1,1\}$ \\
\hline 2 & $\Xi=\{0,0,0,0,0.01, \cdots, 0.99,1,1,1,1\}, \mathcal{H}=\{0,0,0,0,0.02, \cdots, 0.98,1,1,1,1\}$ \\
\hline 3 & $\Xi=\{0,0,0,0,0,0.01, \cdots, 0.99,1,1,1,1,1\}, \mathcal{H}=\{0,0,0,0,0,0.02, \cdots, 0.98,1,1,1,1,1\}$ \\
\hline
\end{tabular}

The optimized designs, including densities at Gauss quadrature points, the 2D view of densities larger than 0.5, the DDF and topology in three cases are displayed in Fig. 18. The numerical results of three cases are all listed in Table 7, consisting of the optimized objective function, the total iterations and volume fraction of the final topology. It can be easily to find that the optimized topologies in three cases are nearly identical. As provided in Table 7, the optimized objective functions in three cases increase with the elevation of the order of the NURBS basis functions, owing to the fact that the high-order IGA elements are usually softer than the lower-order ones [58]. Meanwhile, it is important to note that the total iterative steps are gradually decreased when the order is elevated. The main cause is that the high-order NURBS basis functions can not only exactly capture the geometry, but also improve the computational precision, which can enhance the stability of the optimization. The iteration can quickly arrive at the convergent criterion. Additionally, the volume fractions of the optimized topologies in three cases are nearly equal to $40 \%$.

Table 7. The numerical results in three cases

\begin{tabular}{cc|ccc|ccc}
\hline \multicolumn{2}{c|}{ Case 1 with 2-order } & \multicolumn{2}{c|}{ Case 2 with 3-order } & \multicolumn{2}{c}{ Case 3 with 4-order } \\
\hline Obj Num & Vol & \multicolumn{2}{c|}{ Obj } & Num $\quad$ Vol & Obj & Num Vol \\
\hline
\end{tabular}




\begin{tabular}{lll|lll|lll}
\hline 108.23 & 112 & $40.47 \%$ & 110.39 & 97 & $40.41 \%$ & 114.07 & 72 & $40.12 \%$ \\
\hline
\end{tabular}

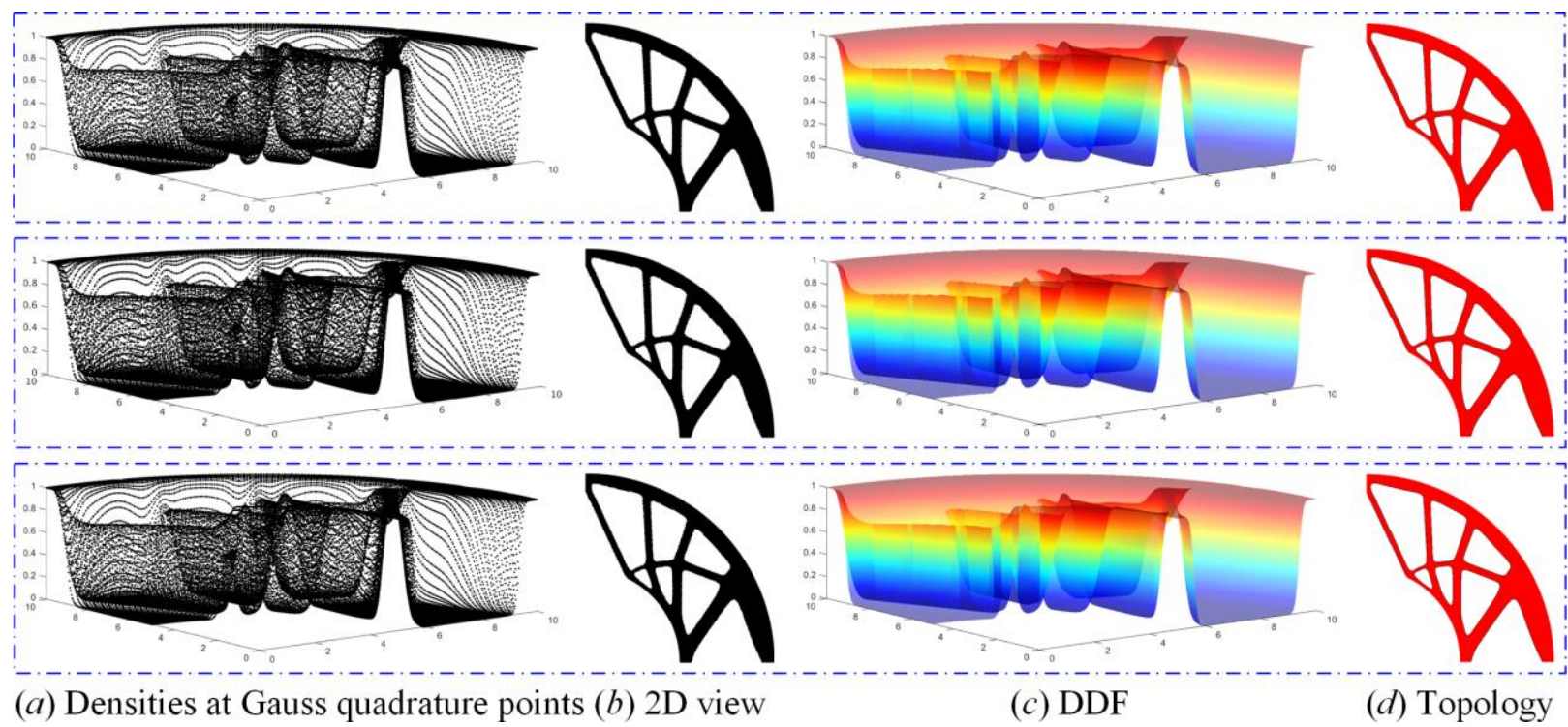

Fig. 18. The optimized designs in three cases

\subsection{Complex structure}

In this section, we present the ability of the developed ITO method to optimize the complex structure. It is difficult to use a single IGA patch to parametrize the geometry, and multiple patches are required. As shown in Fig. 19 (a), a complex structure with the loads and boundary conditions is defined. The structural sizes are defined as: $L=9, H=5$ and $2 r=3.75$. The corresponding IGA mesh with four patches is also displayed in Fig. $19(b)$. The maximum volume fraction is set to be $30 \%$. The initial nodal densities at control points are defined to be 1. The details of the parametrization are listed below Fig. 19.

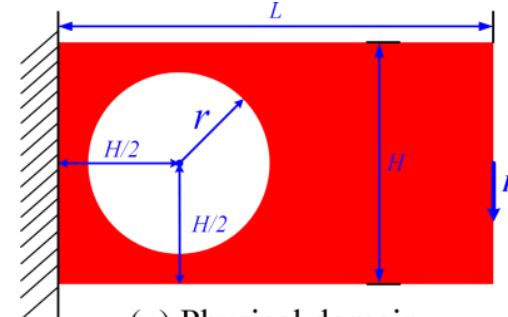

(a) Physical domain

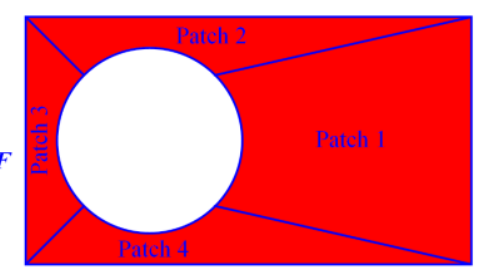

(b) Four patches

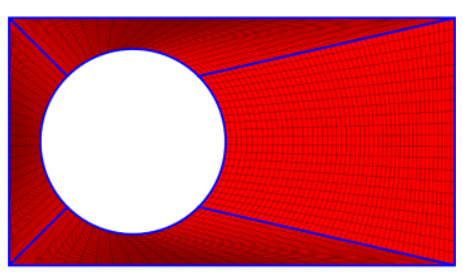

(c) IGA mesh

Fig. 19. Structural design domain: IGA elements $84 \times 49 ; \Xi=\{0,0,0,0,0.0476, \cdots, 0.9524,1,1,1,1\}$,

$$
\mathcal{H}=\{0,0,0,0,0.0204, \cdots, 0.9796,1,1,1,1\} ; p=q=3 .
$$

As shown in Fig. 20, the optimized designs are provided, including densities at Gauss quadrature points, the 2D view of densities larger than 0.5, the DDF and the final topology. Similar to Sections 6.1 and 6.2, the DDF is featured with the sufficiently smoothness and continuity, and the final topology has the smooth structural boundaries and distinct interfaces between the solids and voids. Meanwhile, the iterative histories of the objective and constraint functions are displayed in Fig. 21. It can be easily found that the optimized topology with the maximum stiffness performance can be quickly found by the ITO method in 75 iterations. Hence, the ITO method for the structure parametrized by multiple patches is also featured with the high 
efficiency and perfect stability. Additionally, the volume fraction of the final topology is equal to $30.36 \%$, also nearly equal to the prescribed value $30 \%$, which displays the reasonability of the scheme in Eq. (38).

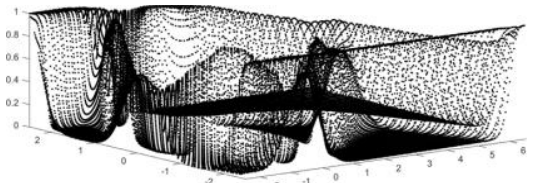

(a) Densities at Gauss quadrature points

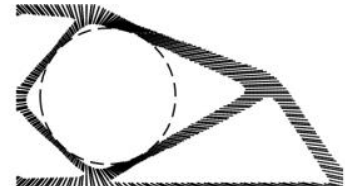

(b) $2 \mathrm{D}$ view

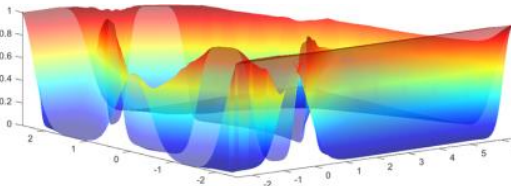

(c) DDF

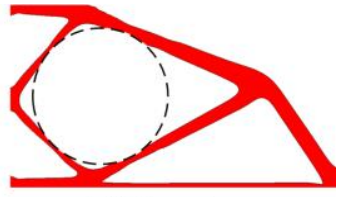

(d) Topology

Fig. 20. The optimized designs

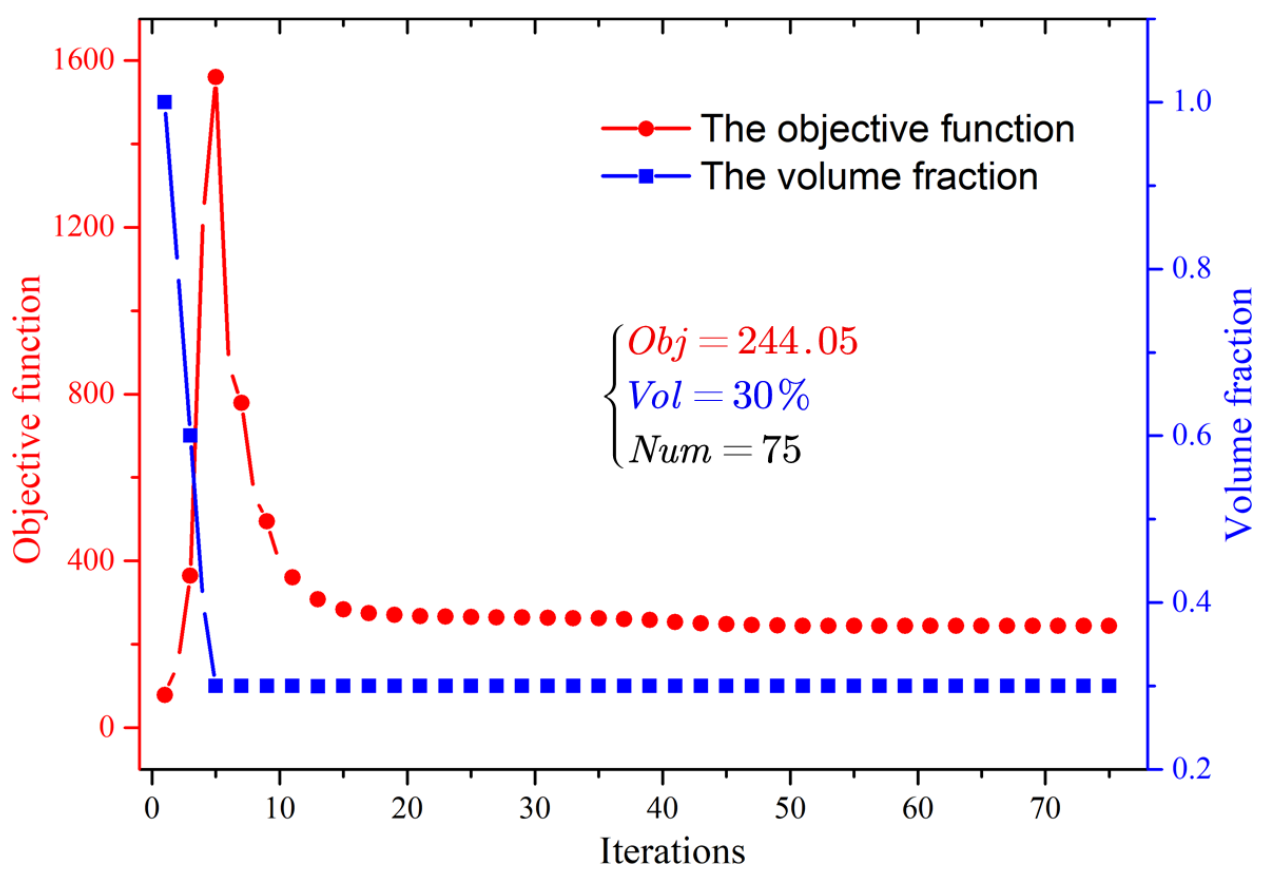

Fig. 21. Convergent histories

\subsection{D Michell structure}

In this section, the ITO method is applied to optimize 3D structures to demonstrate its utility. As shown in Fig. $22(a)$, a 3D Michell structure with the loads and boundary conditions is defined, and the corresponding NURBS solid and the refined IGA mesh are displayed in Fig. $22(b)$ and $(c)$, respectively. The initial model and the coarse IGA mesh is attached in Appendix 1, where the corresponding control points are provided in Table 10. The maximum volume fraction $V_{0}$ is set as $15 \%$, and the initial values of nodal densities are also defined to be 1 .

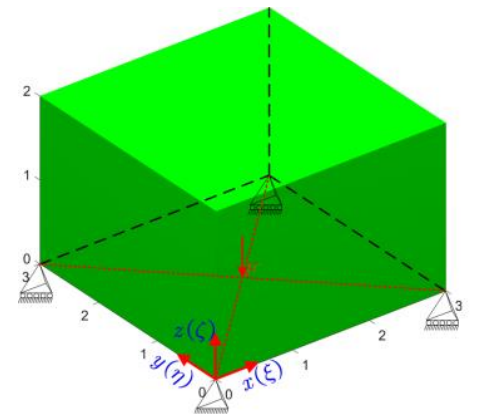

(a) Structural design domain

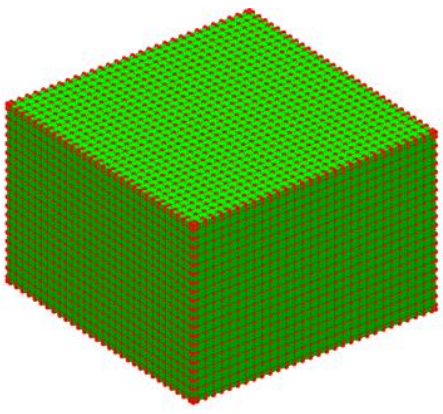

(b) NURBS solid (CAD)

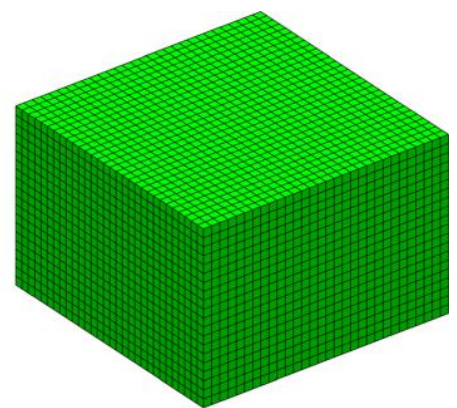

(c) IGA mesh (CAE)

Fig. 22. The 3D Michell structure: IGA elements $30 \times 30 \times 20 ; \Xi=\{0,0,0,0.0333, \cdots, 0.9667,1,1,1\}$, $\mathcal{H}=\{0,0,0,0.0333, \cdots, 0.9667,1,1,1\}, Z=\{0,0,0,0.05, \cdots, 0.95,1,1,1\} ; p=q=r=2$. 
The optimized results of the 3D Michell structure are provided in Table 8. The 3D view of the densities at Gauss quadrature points with the values larger than 0.5 is given in the first column, and the optimized 3D topology is displayed in the second column. In order to display the interior details of the topology, a crosssectional view of the optimized 3D topology is presented in the final column. It is easily to see the optimized 3D topology is still featured with the smooth structural boundaries and distinct interfaces between the solids and voids. The corresponding iterative curves of the objective function, the volume fraction and the change are all shown in Fig. 23. As we can see, the total iterations are equal to 34, which demonstrate the superior efficiency of the ITO method to seek the optimized 3D topology. Meanwhile, the advancing of the change of nodal densities between two consecutive iterations is very stable. The intermediate topologies of the 3D Michell structure are also presented in Fig. 24. Additionally, the volume fraction of the final 3D topology is also calculated, equal to $15.31 \%$ (also nearly $15 \%$ ), which also shows the post-definition scheme for the topology by the DDF is appropriate.

Table 8. The optimized results of 3D Michell structure

3D view of densities

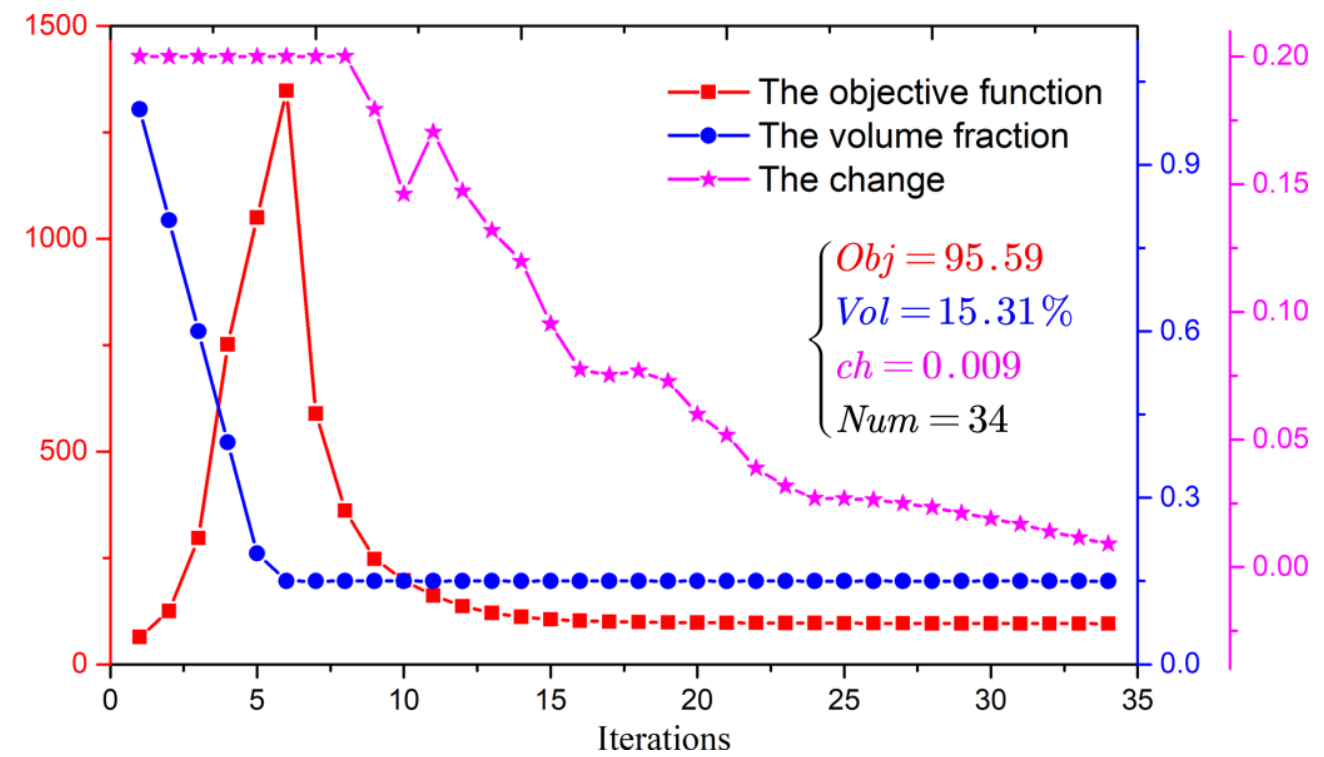

Fig. 23. Convergent histories

Finally, the optimized topology of the 3D Michell structure can be directly outputted in a "STL.file" format and then additively fabricated by the STL technique. The 3D prototypes in three different views are shown 
in Fig. 25. Hence, a systematic procedure for continuum structures with the optimized stiffness performance from the conceptual design stage to the manufacturing phase is realized.

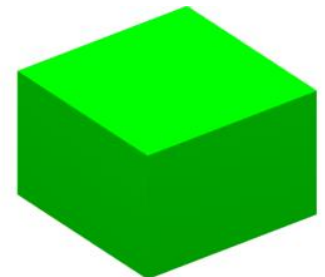

(a) Iteration 1

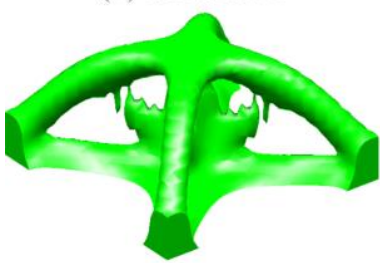

(e) Iteration 18

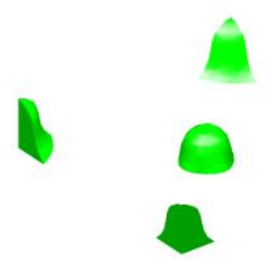

(b) Iteration 6

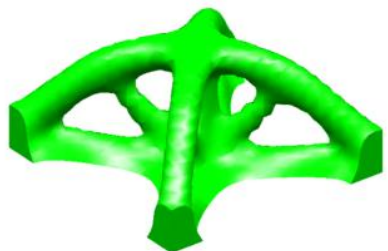

(f) Iteration 25

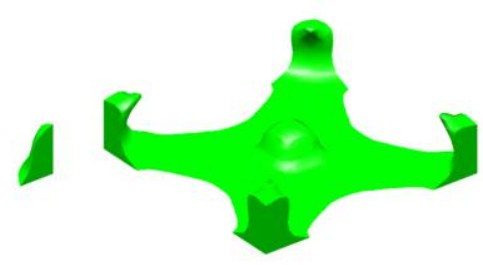

(c) Iteration 9

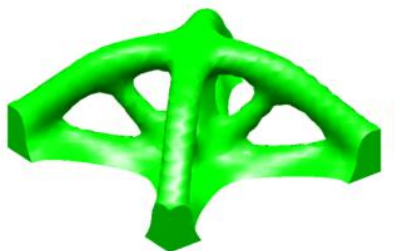

(g) Iteration 30

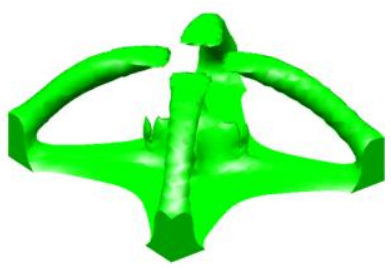

(d) Iteration 12

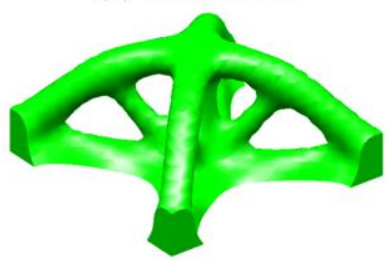

(h) Iteration 34

Fig. 24. The intermediate topologies

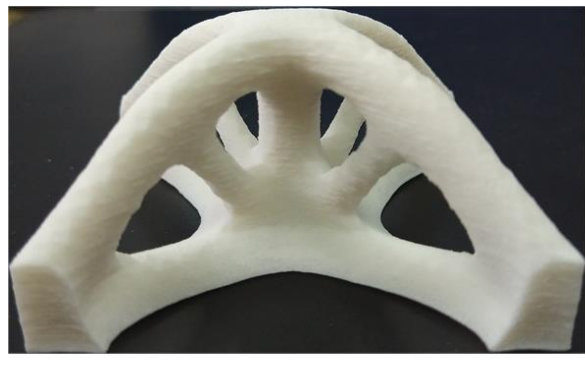

(a) View 1

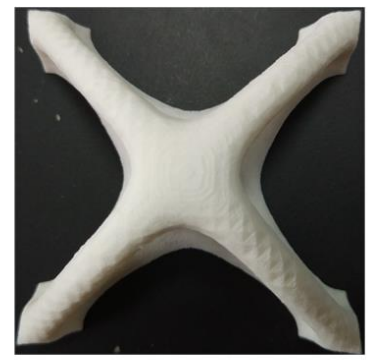

(b) View 2

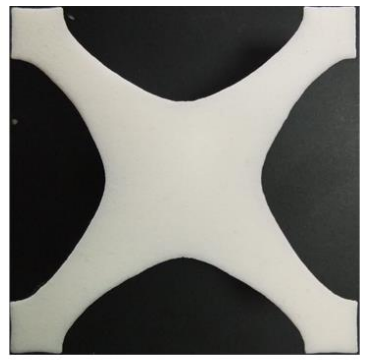

(c) View 3

Fig. 25. 3D printing prototype of the 3D Michell structure

\subsection{D Bridge-type structure}

In this section, a 3D Bridge-type structure with the curved design domain is discussed. As presented in Fig. 26, the design domain with the loads and boundary conditions, the NURBS solid and the IGA mesh are all provided, respectively. The initial geometry model and the coarse IGA mesh are shown in Fig. 31, and the corresponding control points are given in Table 11. The details for the parametrization of the 3D Bridgetype structure are provided below Fig. 26, including the IGA elements, knot vectors and the orders of the NURBS basis functions. The maximum material volume fraction is set as $20 \%$. The initial definition of the nodal densities at control points is also equal to 1 .

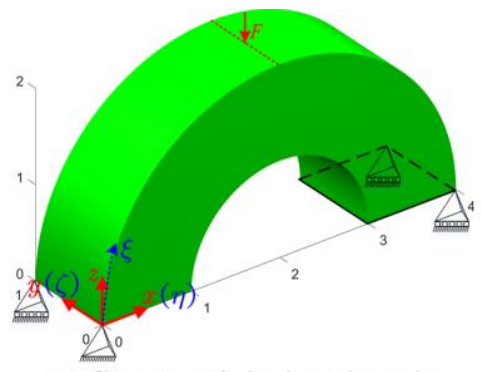

(a) Structural design domain

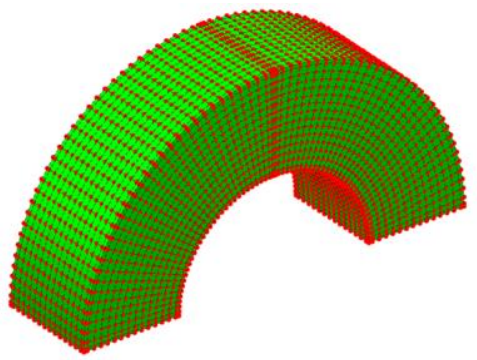

(b) NURBS solid (CAD)

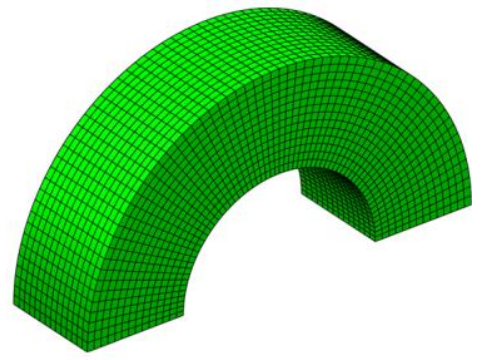

(c) IGA mesh (CAE)

Fig. 26. The 3D Bridge-type structure: IGA elements $64 \times 14 \times 14 ; p=3, q=r=2 ; \Xi=$ $\{0,0,0,0,0.0156, \cdots, 0.4844,0.5,0.5,0.5,0.5156, \cdots, 0.9844,1,1,1,1\}, \mathcal{H}=$
$\{0,0,0,0.0714, \cdots, 0.9286,1,1,1\}, Z=\{0,0,0,0.0714, \cdots, 0.9286,1,1,1\}$. 
As provided in Table 9, the 3D view of densities at Gauss quadrature points with the values larger than 0.5 DGQP, the optimized 3D topology and the corresponding cross-sectional view are displayed. Similarly, the optimized 3D topology also has the smooth structural boundaries and distinct interfaces between the solids and voids, which is beneficial to the latter manufacturing.

Table 9. The optimized results of the 3D Bridge-type structure

3D view of densities

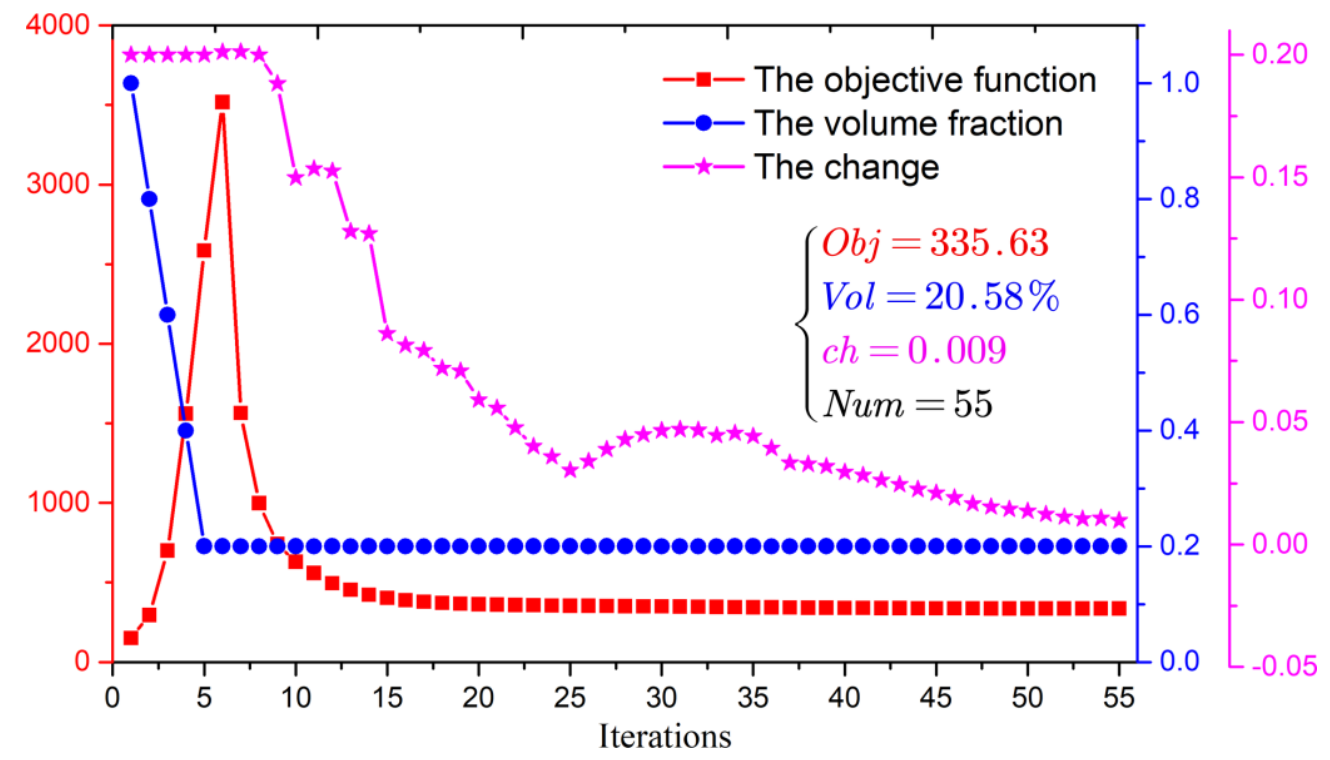

Fig. 27. Convergent histories

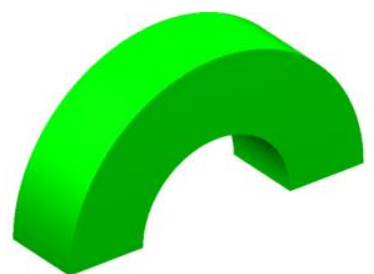

(a) Iteration 1

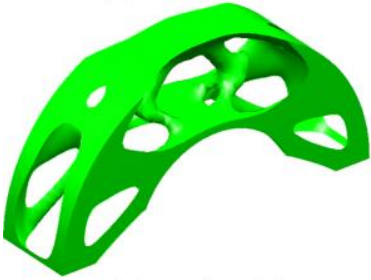

(e) Iteration 18

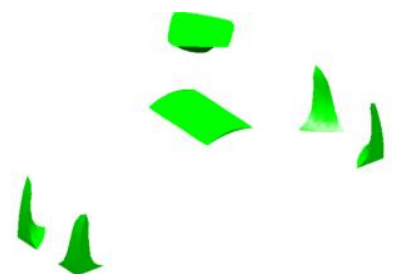

(b) Iteration 6

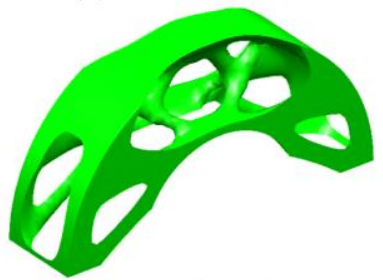

(f) Iteration 25

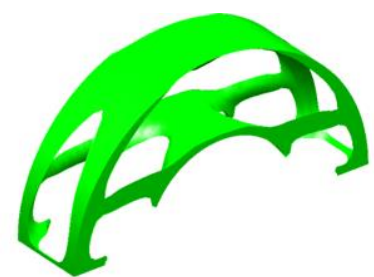

(c) Iteration 9

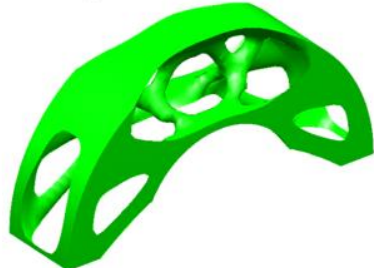

( $g$ ) Iteration 30

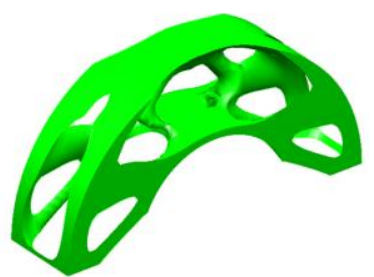

(d) Iteration 12

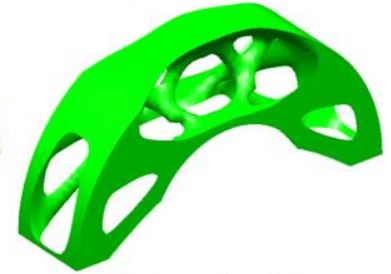

(h) Iteration 34

Fig. 28. The intermediate topologies

As shown in Fig. 27, the iterative curves of the objective function, the volume fraction and the difference of nodal densities between two consecutive steps are also provided. It can be easily found that the optimized 
design can be achieved rapidly within 55 iterations and the convergent curves become plateau afterwards, which shows the high efficiency and superior stability of the ITO method on the optimization of the 3D curved structure. The intermediate topologies in the optimization are also displayed in Fig. 28. A prototype with three views of the optimized 3D topology fabricated by the SLS technique is also shown in Fig. 29.

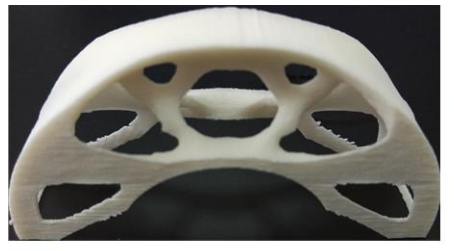

(a) View 1

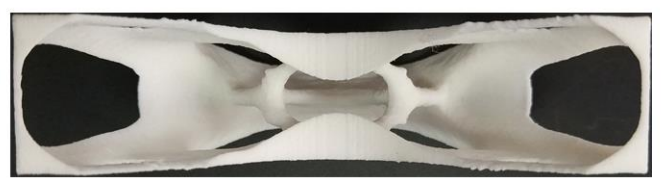

(b) View 2

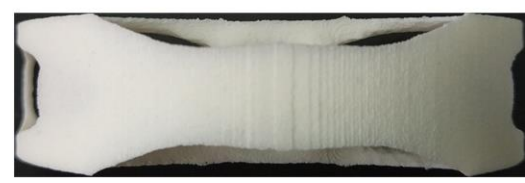

(c) View 3

Fig. 29. 3D Printing prototype of the 3D Bridge-type structure

\section{Conclusions}

In the current work, a more effective and efficient ITO method with the enhanced DDF that has desired smoothness and continuity is proposed for the design of continuum structures. Two procedures are involved in the construction of the DDF, namely the improvement of the overall smoothness of nodal densities and the NURBS parametrization. The ITO formulation is developed for the minimization of the structural mean compliance, where the DDF together with a simple post-processing scheme is employed to represent the topology of the structure, and the IGA is used to evaluate the structural responses.

Several numerical examples in 2D and 3D are discussed to demonstrate the effectiveness and efficiency of the proposed ITO method. It can be easily found that the DDF with the desired smoothness and continuity has a significant influence on the optimization of 2D and 3D structures with the rectangular design domain, the curved shape and the complex geometry. Meanwhile, the final topologies in all numerical examples are featured with smooth boundaries and distinct interfaces between the solids and voids. The optimization of 3D structures presents the effectiveness of the ITO method and the topologically optimized 3D designs are prototyped using the SLS technique.

\section{Appendix}

\section{Appendix 1. Data of the 3D Michell structure}

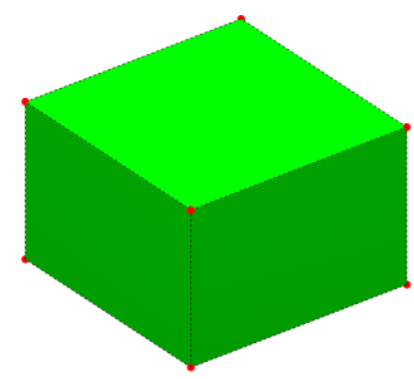

(a) NURBS solid (CAD)

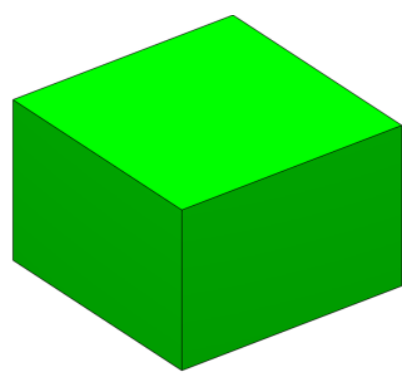

(b) IGA mesh (CAE)

Fig. 30. The 3D Michell structure 


\begin{tabular}{cccccc}
\hline$j$ & $k$ & $\mathbf{P}_{1, j, k}$ & $\mathbf{P}_{2, j, k}$ & $\omega_{1, j, k}$ & $\omega_{2, j, k}$ \\
\hline 1 & 1 & $(0,0,0)$ & $(3,0,0)$ & 1 & 1 \\
2 & 1 & $(0,3,0)$ & $(3,3,0)$ & 1 & 1 \\
1 & 2 & $(0,0,2)$ & $(3,0,2)$ & 1 & 1 \\
2 & 2 & $(0,3,2)$ & $(3,3,2)$ & 1 & 1 \\
\hline
\end{tabular}

\section{Appendix 2. Data of the 3D Bridge-type structure}

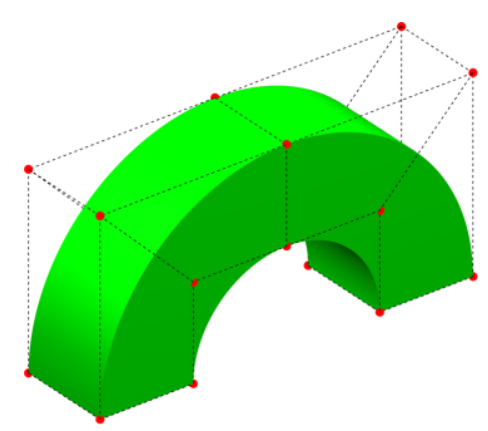

(a) NURBS solid (CAD)

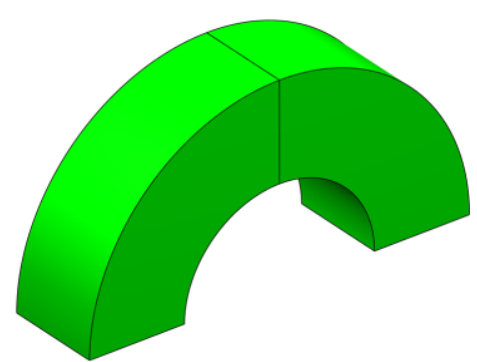

(b) IGA mesh (CAE)

Fig. 31. The 3D Bridge-type structure

Table 11. Control points of the 3D Bridge-type structure

\begin{tabular}{cccccc}
\hline$i$ & $j$ & $\mathbf{P}_{i, j, 1}$ & $\mathbf{P}_{i, j, 2}$ & $\omega_{i, j, 1}$ & $\omega_{i, j, 2}$ \\
\hline 1 & 1 & $(0,0,0)$ & $(0,1,0)$ & 1 & 1 \\
2 & 1 & $(0,0, \sqrt{2})$ & $(0,1 / \sqrt{2}, \sqrt{2})$ & $1 / \sqrt{2}$ & $1 / \sqrt{2}$ \\
3 & 1 & $(2,0,2)$ & $(2,1,2)$ & 1 & 1 \\
4 & 1 & $(2 \sqrt{2}, 0, \sqrt{2})$ & $(2 \sqrt{2}, 1 / \sqrt{2}, \sqrt{2})$ & $1 / \sqrt{2}$ & $1 / \sqrt{2}$ \\
5 & 1 & $(4,0,0)$ & $(4,1,0)$ & 1 & 1 \\
1 & 2 & $(1,0,0)$ & $(1,1,0)$ & 1 & 1 \\
2 & 2 & $(1 / \sqrt{2}, 0,1 / \sqrt{2})$ & $(1 / \sqrt{2}, 1 / \sqrt{2}, 1 / \sqrt{2})$ & $1 / \sqrt{2}$ & $1 / \sqrt{2}$ \\
3 & 2 & $(2,0,1)$ & $(2,1,1)$ & 1 & 1 \\
4 & 2 & $(3 / \sqrt{2}, 0,1 / \sqrt{2})$ & $(3 / \sqrt{2}, 1 / \sqrt{2}, 1 / \sqrt{2})$ & $1 / \sqrt{2}$ & $1 / \sqrt{2}$ \\
5 & 2 & $(3,0,0)$ & $(3,1,0)$ & 1 & 1 \\
\hline
\end{tabular}

\section{Acknowledgments}

This work was partially supported by the Australian Research Council (ARC) - Discovery Projects (160102491), and the China Equipment Pre-research Program (41423010102), and the National Basic Scientific Research Program of China (JCKY2016110C012).

\section{References}

1. Bendsøe MP, Sigmund O. Topology Optimization: Theory, Methods and Applications. 2003. 
2. Bendsøe MP, Kikuchi N. Generating optimal topologies in stuctural design using a homogenization method. Comput Methods Appl Mech Eng 1988; 71(2):197-224.

3. Zhou M, Rozvany GIN. The COC algorithm, Part II: Topological, geometrical and generalized shape optimization. Computer Methods in Applied Mechanics and Engineering 1991; 89(1-3):309-336.

4. Bendsøe MP, Sigmund O. Material interpolation schemes in topology optimization. Archive of Applied Mechanics 1999; 69(9-10):635-654.

5. Xie YM, Steven GP. A simple evolutionary procedure for structural optimization. Computers and Structures 1993; 49(5):885-969.

6. Sethian JA, Wiegmann A. Structural Boundary Design via Level Set and Immersed Interface Methods. Journal of Computational Physics 2000; 163(2):489-528.

7. Wang MY, Wang X, Guo D. A level set method for structural topology optimization. Computer Methods in Applied Mechanics and Engineering 2003; 192(1-2):227-246.

8. Allaire G, Jouve F, Toader AM. Structural optimization using sensitivity analysis and a level-set method. Journal of Computational Physics 2004; 194(1):363-393.

9. Guo X, Zhang W, Zhong W. Doing Topology Optimization Explicitly and Geometrically -A New Moving Morphable Components Based Framework. Journal of Applied Mechanics 2014; 81(8):081009.

10. Guo X, Zhang W, Zhang J, Yuan J. Explicit structural topology optimization based on moving morphable components (MMC) with curved skeletons. Computer Methods in Applied Mechanics and Engineering 2016; 310:711-748.

11. Du J, Olhoff N. Topological design of freely vibrating continuum structures for maximum values of simple and multiple eigenfrequencies and frequency gaps. Structural and Multidisciplinary Optimization 2007; 34(2):91-110.

12. Ma Z-D, Kikuchi N, Hagiwara I. Structural topology and shape optimization for a frequency response problem. Computational Mechanics 1993; 13(3):157-174.

13. Zhang W, Liu H, Gao T. Topology optimization of large-scale structures subjected to stationary random excitation: An efficient optimization procedure integrating pseudo excitation method and mode acceleration method. Computers \& Structures 2015; 158:61-70.

14. Sigmund O. Materials with prescribed constitutive parameters: An inverse homogenization problem. International Journal of Solids and Structures 1994; 31(17):2313-2329.

15. Guest JK, Prévost JH. Optimizing multifunctional materials: Design of microstructures for maximized stiffness and fluid permeability. International Journal of Solids and Structures 2006.

16. Gao J, Li H, Gao L, Xiao M. Topological shape optimization of 3D micro-structured materials using energy-based homogenization method. Advances in Engineering Software 2018; 116:89-102.

17. Rodrigues H, Guedes JM, Bendsoe MP. Hierarchical optimization of material and structure. Structural and Multidisciplinary Optimization 2002; 24(1):1-10.

18. Xia L, Breitkopf P. Concurrent topology optimization design of material and structure within FE2 nonlinear multiscale analysis framework. Computer Methods in Applied Mechanics and Engineering 2014; 278:524542.

19. Yan J, Duan Z, Lund E, Wang J. Concurrent multi-scale design optimization of composite frames with manufacturing constraints. Structural and Multidisciplinary Optimization 2017; 56(3):519-533.

20. Groen JP, Sigmund O. Homogenization-based topology optimization for high-resolution manufacturable microstructures. International Journal for Numerical Methods in Engineering 2018; 113(8):1148-1163.

21. Li H, Luo Z, Gao L, Qin Q. Topology optimization for concurrent design of structures with multi-patch microstructures by level sets. Computer Methods in Applied Mechanics and Engineering 2018; 331:536561. 
22. Nishi S, Terada K, Kato J, Nishiwaki S, Izui K. Two-scale topology optimization for composite plates with in-plane periodicity. International Journal for Numerical Methods in Engineering 2018; 113(8):1164-1188.

23. Gao J, Luo Z, Li H, Gao L. Topology optimization for multiscale design of porous composites with multidomain microstructures. Computer Methods in Applied Mechanics and Engineering 2019; 344:451-476.

24. Gao J, Luo Z, Li H, Li P, Gao L. Dynamic multiscale topology optimization for multi-regional microstructured cellular composites. Composite Structures 2019; 211:401-417.

25. Chu S, Gao L, Xiao M, Luo Z, Li H, Gui X. A new method based on adaptive volume constraint and stress penalty for stress-constrained topology optimization. Structural and Multidisciplinary Optimization 2018; 57(3):1163-1185.

26. Chu S, Gao L, Xiao M, Luo Z, Li H. Stress-based multi-material topology optimization of compliant mechanisms. International Journal for Numerical Methods in Engineering 2018; 113(7):1021-1044.

27. Zhang W, Zhao L, Gao T, Cai S. Topology optimization with closed B-splines and Boolean operations. Computer Methods in Applied Mechanics and Engineering 2017; 315:652-670.

28. Díaz A, Sigmund O. Checkerboard patterns in layout optimization. Structural optimization 1995; 10(1):4045.

29. Sigmund O, Petersson J. Numerical instabilities in topology optimization: A survey on procedures dealing with checkerboards, mesh-dependencies and local minima. Structural optimization 1998; 16(1):68-75.

30. Sigmund O. Morphology-based black and white filters for topology optimization. Structural and Multidisciplinary Optimization 2007; 33(4-5):401-424.

31. Matsui K, Terada K. Continuous approximation of material distribution for topology optimization. International Journal for Numerical Methods in Engineering 2004; 59(14):1925-1944.

32. Rahmatalla SF, C.C. S. A Q4/Q4 continuum structural topology optimization implementation. Structural and Multidisciplinary Optimization 2004; 27(1):130-135.

33. Paulino GH, Le CH. A modified Q4/Q4 element for topology optimization. Structural and Multidisciplinary Optimization 2009; 37(3):255-264.

34. Guest JK, Prévost JH, Belytschko T. Achieving minimum length scale in topology optimization using nodal design variables and projection functions. International Journal for Numerical Methods in Engineering 2004; 61(2):238-254.

35. Kang Z, Wang Y. Structural topology optimization based on non-local Shepard interpolation of density field. Computer Methods in Applied Mechanics and Engineering 2011; 200(49-52):3515-3525.

36. Kang Z, Wang Y. A nodal variable method of structural topology optimization based on Shepard interpolant. International Journal for Numerical Methods in Engineering 2012; 90(3):329-342.

37. Luo Z, Zhang N, Wang Y, Gao W. Topology optimization of structures using meshless density variable approximants. International Journal for Numerical Methods in Engineering 2013; 93(4):443-464.

38. Hughes TJRJR, Cottrell JAA, Bazilevs Y. Isogeometric analysis: CAD, finite elements, NURBS, exact geometry and mesh refinement. Computer Methods in Applied Mechanics and Engineering 2005; 194(3941):4135-4195.

39. Cottrell JA, Hughes TJR, Bazilevs Y. Isogeometric Analysis: Toward Integration of CAD and FEA.; 2009.

40. Nguyen VP, Anitescu C, Bordas SPA, Rabczuk T. Isogeometric analysis: An overview and computer implementation aspects. Mathematics and Computers in Simulation 2015; 117:89-116.

41. Seo Y-D, Kim H-J, Youn S-K. Isogeometric topology optimization using trimmed spline surfaces. Computer Methods in Applied Mechanics and Engineering 2010; 199(49-52):3270-3296.

42. Dedè L, Borden MJ, Hughes TJR. Isogeometric Analysis for Topology Optimization with a Phase Field Model. Archives of Computational Methods in Engineering 2012; 19(3):427-465.

43. Hassani B, Khanzadi M, Tavakkoli SM. An isogeometrical approach to structural topology optimization by optimality criteria. Structural and Multidisciplinary Optimization 2012; 45(2):223-233. 
44. Hassani B, Tavakkoli SM, Ghasemnejad H. Simultaneous shape and topology optimization of shell structures. Structural and Multidisciplinary Optimization 2013; 48(1):221-233.

45. Kang P, Youn S-K. Isogeometric topology optimization of shell structures using trimmed NURBS surfaces. Finite Elements in Analysis and Design 2016; 120:18-40.

46. Qian X. Topology optimization in B-spline space. Computer Methods in Applied Mechanics and Engineering 2013; 265:15-35.

47. Luo Z, Wang MY, Tong L, Wang S. Shape and topology optimization of compliant mechanisms using a parameterization level set method. Journal of Computational Physics 2007; 227(1):680-705.

48. Luo Z, Wang M, Wang S, Wei P. A level set based parameterization method for structural shape and topology optimization. International Journal for Numerical Methods in Engineering 2008; 76(1):1-26.

49. Wang Y, Benson DJ. Isogeometric analysis for parameterized LSM-based structural topology optimization. Computational Mechanics 2016; 57(1):19-35.

50. Jahangiry HA, Tavakkoli SM. An isogeometrical approach to structural level set topology optimization. Computer Methods in Applied Mechanics and Engineering 2017; 319:240-257.

51. Liu H, Yang D, Hao P, Zhu X. Isogeometric analysis based topology optimization design with global stress constraint. Computer Methods in Applied Mechanics and Engineering 2018; 342:625-652.

52. Ghasemi H, Park HS, Rabczuk T. A level-set based IGA formulation for topology optimization of flexoelectric materials. Computer Methods in Applied Mechanics and Engineering 2017; 313:239-258.

53. Lieu QX, Lee J. Multiresolution topology optimization using isogeometric analysis. International Journal for Numerical Methods in Engineering 2017; 112(13):2025-2047.

54. Piegl L, Tiller W. The NURBS book. Springer Science \& Business Media; 2012.

55. Shepard D. A two-dimensional interpolation function for irregularly-spaced data. In: Proceedings of the 1968 23rd ACM national conference. ACM; 1968:517-524.

56. Wendland H. Piecewise polynomial, positive definite and compactly supported radial functions of minimal degree. Advances in computational Mathematics 1995; 4(1):389-396.

57. Rozvany GIN, Bendsøe MP, Kirsch U. Layout optimization of structures. Applied Mechanics Reviews 1995; 48(2):41-119.

58. Hughes TJR. The finite element method: linear static and dynamic finite element analysis. Courier Corporation; 2012. 